\title{
Macrophages regulate corpus luteum development during embryo implantation in mice
}

\author{
Alison S. Care, ${ }^{1}$ Kerrilyn R. Diener, ${ }^{1,2,3}$ Melinda J. Jasper, ${ }^{1}$ Hannah M. Brown, ${ }^{1}$ \\ Wendy V. Ingman, ${ }^{1,4}$ and Sarah A. Robertson 1 \\ ${ }^{1}$ Robinson Institute and School of Paediatrics and Reproductive Health, University of Adelaide, Adelaide, Australia. ${ }^{2}$ Experimental Therapeutics Laboratory, \\ Hanson Institute, Adelaide, Australia. ${ }^{3}$ Sansom Institute, School of Pharmacy and Medical Sciences, University of South Australia, Adelaide, Australia. \\ ${ }^{4}$ School of Medicine, Queen Elizabeth Hospital, University of Adelaide, Woodville, Australia.
}

\begin{abstract}
Macrophages are prominent in the uterus and ovary at conception. Here we utilize the Cd11b-Dtr mouse model of acute macrophage depletion to define the essential role of macrophages in early pregnancy. Macrophage depletion after conception caused embryo implantation arrest associated with diminished plasma progesterone and poor uterine receptivity. Implantation failure was alleviated by administration of bone marrowderived $\mathrm{CD} 11 \mathrm{~b}^{+} \mathrm{F} 4 / \mathrm{80}^{+}$monocytes/macrophages. In the ovaries of macrophage-depleted mice, corpora lutea were profoundly abnormal, with elevated Ptgs2, Hifla, and other inflammation and apoptosis genes and with diminished expression of steroidogenesis genes Star, Cyp11a1, and Hsd3b1. Infertility was rescued by exogenous progesterone, which confirmed that uterine refractoriness was fully attributable to the underlying luteal defect. In normally developing corpora lutea, macrophages were intimately juxtaposed with endothelial cells and expressed the proangiogenic marker TIE2. After macrophage depletion, substantial disruption of the luteal microvascular network occurred and was associated with altered ovarian expression of genes that encode vascular endothelial growth factors. These data indicate a critical role for macrophages in supporting the extensive vascular network required for corpus luteum integrity and production of progesterone essential for establishing pregnancy. Our findings raise the prospect that disruption of macrophage-endothelial cell interactions underpinning corpus luteum development contributes to infertility in women in whom luteal insufficiency is implicated.
\end{abstract}

\section{Introduction}

Macrophages have a broad range of functions including phagocytosis, antigen presentation, and secretion of a variety of cytokines, growth factors, and tissue-remodeling agents (1). Their central role in the generation and execution of immune responses is undisputed. More recently, additional roles have emerged for macrophages in tissue development and homeostasis, including angiogenesis and vascular remodeling, in embryogenesis as well as in the adult organism (2-4). There is considerable heterogeneity and plasticity in macrophage phenotypes, and their differentiation and function within tissues is influenced by local environmental signals that direct release of various cytokines, growth factors, and tissue-remodeling agents in tissue-specific phenotypes $(1-3,5)$.

A developmental function of macrophages is particularly evident in female reproductive tract tissues that mature after birth and thereafter undergo repeated cycles of breakdown, repair, and regeneration $(6,7)$. In the uterus, macrophages fluctuate in number and phenotype over the ovarian cycle, and additional macrophages are recruited into the endometrium in an inflammationlike response to seminal fluid during the pre-implantation period of early pregnancy $(8,9)$. Uterine macrophages are implicated in remodeling processes and in inducing expression of epithelial

Authorship note: Kerrilyn R. Diener and Melinda J. Jasper contributed equally to this work.

Conflict of interest: The authors have declared that no conflict of interest exists. Citation for this article: J Clin Invest. 2013;123(8):3472-3487. doi:10.1172/JCI60561 glycoproteins associated with embryo attachment (10), and they potentially contribute to subsequent events of the uterine decidual response and placental trophoblast invasion (11). Within the ovary, macrophages accumulate within the thecal layer of developing follicles, and there is evidence for a supporting role in follicle growth and ovulation (12-14) as well as for a contribution to vascular homeostasis (15). Immediately after ovulation, macrophages migrate into the developing corpora lutea in the mouse (16), rat (17), and human ovaries (18). There they are implicated in influencing the steroidogenic function of luteal cells as well as remodeling after luteal regression $(19,20)$.

These observations raise the question of the importance of macrophage contributions to the sequence of events in reproductive tissues necessary for embryo implantation and initiation of pregnancy. Implantation depends on an adequately prepared endometrium to permit embryo attachment, allow uterine transformation in the decidual response, and facilitate invasion of placental cells. In turn, uterine receptivity is regulated by corpus luteum steroidogenic function to produce sufficient ovarian steroid hormones, progesterone, and estrogen. Although the presence of macrophages in uterine and ovarian tissues suggests a role in these events, their absolute necessity for pregnancy has not been formally evaluated.

Studies in the osteopetrotic Csf1 $\mathrm{op} / \mathrm{Cs} f 1^{\text {op }}$ mouse (which has a naturally occurring Csf1-null mutation) have provided some insight into the functional role of macrophages in reproduction. M-CSF (also referred to as CSF-1) stimulates macrophage proliferation, differentiation, and recruitment, and Csf1 ${ }^{\text {op }} / \mathrm{Cs} f 1^{\circ \mathrm{p}}$ mice are 
A

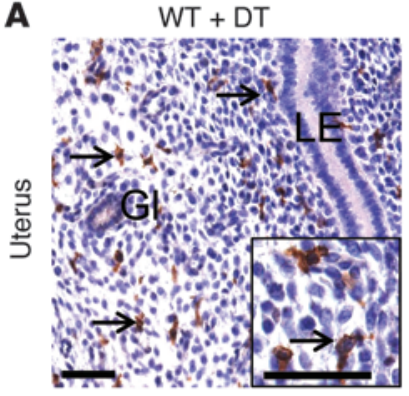

B

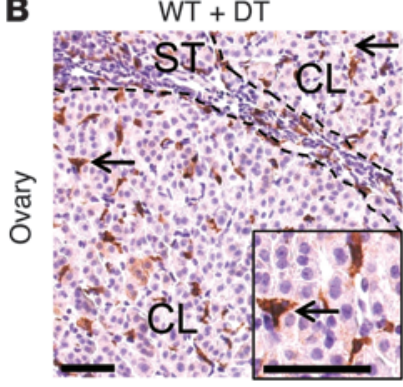

$C d 11 b^{-}+\mathrm{DT}$

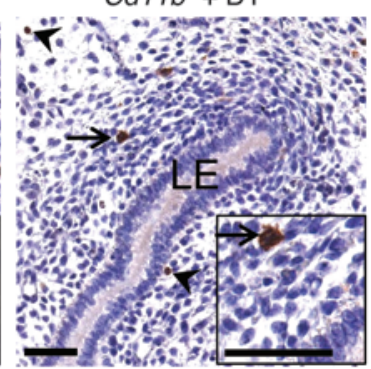

$C d 11 b^{-}+$DT

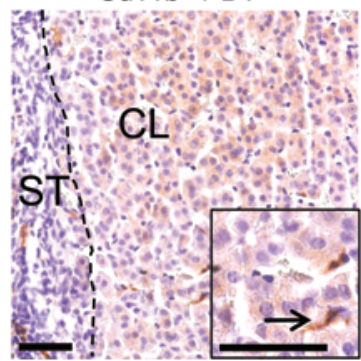

(5)

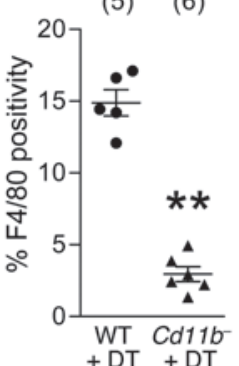

(5) (3)

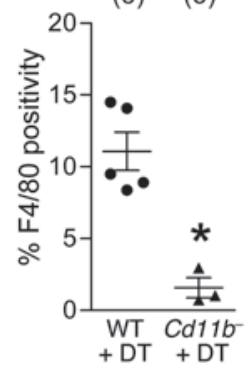

C
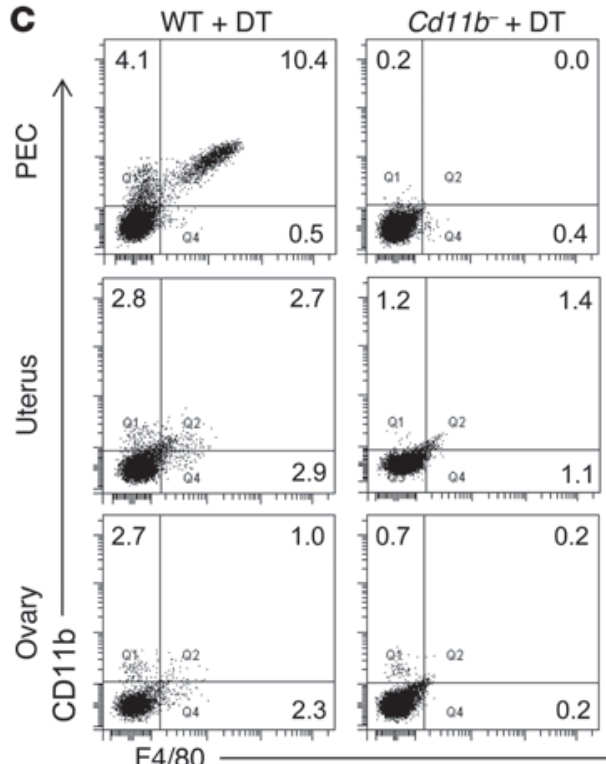
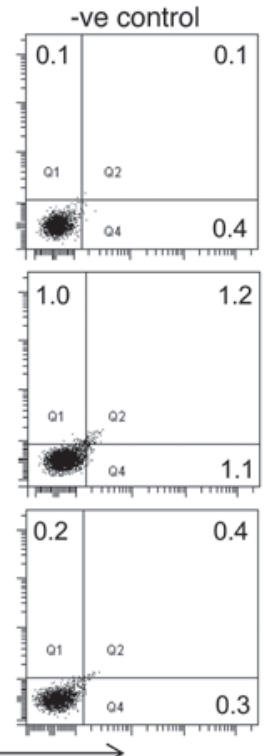

\section{Figure 1}

DT administration to Cd11b-Dtr mice elicits macrophage depletion in the uterus and ovary. Tissues were recovered from wild-type control or Cd11b-Dtr mice on day $4.5 \mathrm{pc}, 24$ hours after i.p. injection of DT $(25 \mathrm{ng} / \mathrm{g})$. (A and $\mathbf{B}$ ) Sections of uterus (A) and ovary (B) labeled with anti-F4/80 indicated few macrophages (arrows) remaining in the uterus and ovary of Cd11b-Dtr mice (right panels; insets are high power) compared with wild-type mice (left panels). Some uterine $\mathrm{F} 4 / 80^{+}$cells were eosinophils (arrowheads), and these were retained after DT treatment (see Supplemental Figure 1). The percent positivity for $\mathrm{F} 4 / 80^{+}$cells is shown for wild-type mice (WT +DT) and macrophage-depleted Cd11b-Dtr mice (Cd11b+ DT) administered DT. Ten individual fields per uterus were analyzed, and the number of mice per group is indicated in parentheses. LE, luminal epithelium; GI, uterine gland; CL, corpus luteum; ST, stroma. Scale bars: $50 \mu \mathrm{m}$. (C) Single-cell suspensions recovered from the peritoneal cavity (PEC) or prepared by enzymatic digestion of uterus and ovary were analyzed using FACS with anti-CD11b and anti-F4/80 antibodies, and show substantially diminished CD11b+F4/80+ macrophages in Cd11b-Dtr mice compared with wildtype controls. FACS plots are representative of 4-6 mice per group. Quantitative FACS data are given in Supplemental Table 1. ${ }^{\star} P<0.05 ;{ }^{* \star} P<0.01$. severely depleted of macrophages in many tissues (21). In the nonpregnant cycling state, the ovary $(7,16)$ and uterus $(22)$ are almost completely devoid of macrophages. Csf1 $\mathrm{op} / \mathrm{Cs} f 1^{\text {op }}$ exhibit extended estrous cycles and a lower ovulation rate, which are attributed to impaired function of the hypothalamic-pituitary-gonadal axis as well as intra-ovarian defects $(16,23)$.

However, Csf1-null mutant mice have limited utility for interrogating macrophage functions in establishing and maintaining pregnancy, since after mating, cytokines and chemokines other than M-CSF attract macrophages into the ovary and uterus, and pregnancy progresses normally albeit in a reduced proportion of animals $(16,22)$. On day 1 post-coitum (pc), the density of $\mathrm{F} 4 / 80^{+}$ macrophages in the uterus of Csf1-null mutant mice is approximately $53 \%$ that of wild-type mice $(24,25)$, while in corpora lutea, $35 \%$ of the normal density of $\mathrm{F} 4 / 80^{+}$macrophages is attained (16).
The Cd11b-Dtr transgenic mouse (26) provides a new experimental approach to examine the role of macrophages in reproductive processes. This model allows acute, systemic depletion of CD $11 \mathrm{~b}^{+}$ macrophages following the administration of nanogram quantities of diphtheria toxin (DT). Here we report that acute depletion of $\mathrm{CD}_{11} \mathrm{~b}^{+}$cells during early pregnancy results in complete implantation failure, and that this is alleviated by administration of DT-resistant wild-type macrophages. The corpora lutea of macrophage-depleted mice produce substantially less progesterone, have disrupted blood vasculature, and exhibit changes in the local expression of genes encoding angiogeneic regulators. The reduced progesterone production accounts fully for the infertility defect, since pregnancy can be restored and supported to term through exogenous progesterone administration. These findings demonstrate that in the mouse, macrophages with a TIE2+ proangiogenic 
A DT at day $0.5 \mathrm{pc}$

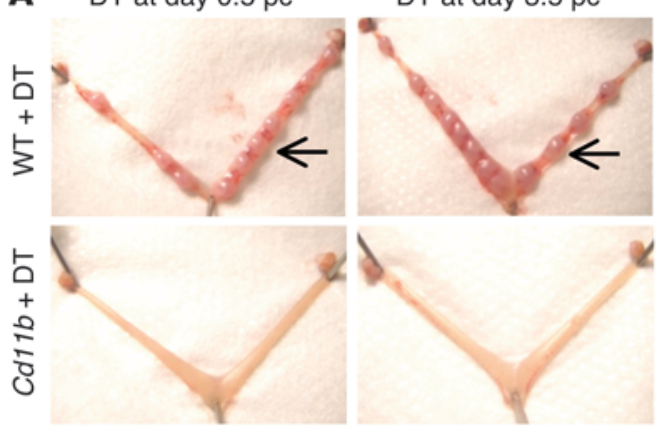

DT at day $3.5 \mathrm{pc}$

B $\quad \mathrm{Cd}_{11 b^{-}}+\mathrm{DT}+\mathrm{BM}$

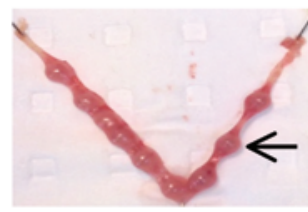

C $\quad C d 11 b^{-}+\mathrm{GI} 52-\mathrm{DT}$
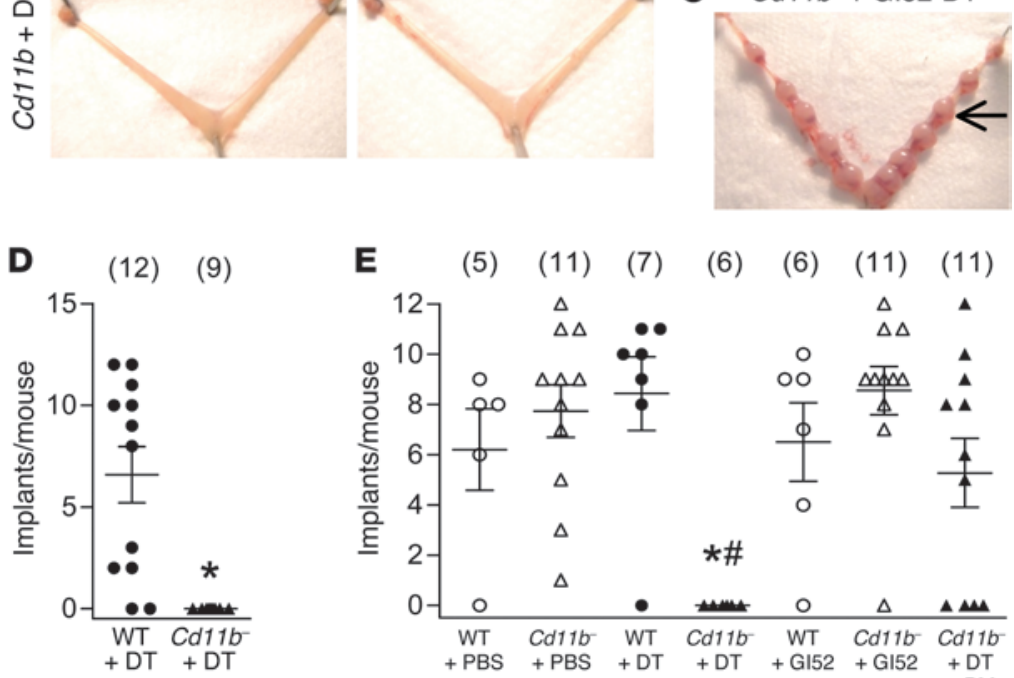

E

(5)

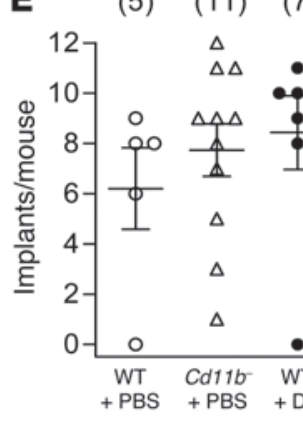

(7)

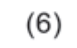

6)

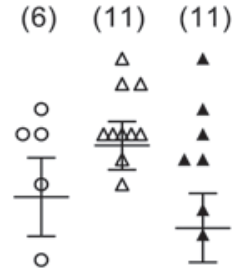

phenotype have an essential role in orchestrating the neovascularization pivotal to corpus luteum development, progesterone synthesis, and the establishment of viable pregnancy.

\section{Results}

Macrophage depletion during the pre-implantation period causes pregnancy failure. To evaluate the utility of Cd11b-Dtr mice as a model for macrophage depletion from reproductive tissues during early pregnan$c y$, the ovaries and uterus were dissected on day $4.5 \mathrm{pc}$ (when embryo implantation commences), 24 hours after DT injection on day 3.5 pc, and analyzed using immunohistochemistry for the macrophagespecific marker F4/80. Uterine macrophages were depleted by $84 \%$ in DT-treated Cd11b-Dtr mice compared with DT-treated wild-type control mice, in which macrophages were present throughout the endometrial stroma, particularly subjacent to the luminal epithelial (Figure 1A). In the ovaries of DT-treated Cd11b-Dtr mice, macrophages were depleted by $82 \%$ compared with wild-type mice, in which they were distributed throughout the ovarian stroma, within corpora lutea, and in the theca surrounding follicles (Figure 1B). Similar proportions of macrophages were removed from the uterus and ovaries when DT was given at day $0.5 \mathrm{pc}$, and tissues were evaluated 24 hours later (data not shown). Of the small numbers of $\mathrm{F} 4 / 80^{+}$cells retained in the uterus after DT treatment, most were eosinophils (Supplemental Figure 1; supplemental material available online with this article; doi:10.1172/JCI60561DS1), which are present in the uterus (27) but not the ovary (13).

When single-cell suspensions from uterus and ovary were evaluated using flow cytometry for CD11b and F4/80 expression (Figure 1C and Supplemental Table 1), a similar extent of macrophage depletion was seen. Macrophages in the peritoneal cavity were almost completely ablated after DT administration to Cd11b-Dtr mice, as reported by others $(26,28)$.

\section{Figure 2}

Macrophage depletion during the pre-implantation period causes complete infertility in Cd11b-Dtr mice. (A) At autopsy on day $7.5 \mathrm{pc}$, macrophage-depleted Cd11b-Dtr mice had no visible implantation sites (lower panels) after injection with DT $(25 \mathrm{ng} / \mathrm{g})$ on day $0.5 \mathrm{pc}$ or day $3.5 \mathrm{pc}$, compared with normal implantation sites (arrows) in wildtype mice given DT (upper panels). (B) Normal implantation sites (arrows) were seen when Cd11b-Dtr mice were reconstituted with wild-type bone marrow-derived $\mathrm{CD} 11 \mathrm{~b}^{+} \mathrm{F} 4 / 80^{+}$monocytes (Supplemental Figure 2) prior to DT injection on day $3.5 \mathrm{pc}(\mathrm{Cd} 11 \mathrm{~b}-+\mathrm{DT}+\mathrm{BM})$. (C) Normal implantation sites (arrows) were also seen when Cd11b-Dtr mice were injected with biologically inactive [Glu52]-DT (Gl52-DT). (D) The number of implantation sites per mouse is shown for wild-type mice (WT +DT) and macrophage-depleted Cd11b-Dtr mice (Cd11b- +DT) administered DT on day $0.5 \mathrm{pc}$. (E) The number of implantation sites per mouse is shown for wild-type mice and Cd11b-Dtr mice administered PBS, DT or [Glu52]-DT on day $3.5 \mathrm{pc}$, or DT on day $3.5 \mathrm{pc}$ after reconstitution with wild-type bone marrow-derived $\mathrm{CD} 11 \mathrm{~b}+\mathrm{F} 4 / 80^{+}$monocytes (Cd11b-+DT +BM). Data are number of implantations per mouse, with mean \pm SEM superimposed. The number of mice in each group is shown in parentheses. ${ }^{*} P<0.01$, Cd11b- +DT versus WT +DT; $P<<0.01, C d 11 b-+\mathrm{DT}+\mathrm{BM}$ versus Cd11b- +DT.
To investigate the effect of macrophage depletion during the pre-implantation period on pregnancy progression, DT was administered on day $0.5 \mathrm{pc}$ or $3.5 \mathrm{pc}$ to Cd11b-Dtr and wild-type mice. When treated mice were autopsied at day $7.5 \mathrm{pc}$, complete pregnancy failure was evident (Figure $2 \mathrm{~A}$ ), and similar results were obtained if DT was given on days $1.5 \mathrm{pc}$ or $2.5 \mathrm{pc}$ (data not shown). In contrast, healthy implantation sites were seen in the majority of wild-type mice given DT (Figure 2, A, D, and E). Additional control groups of Cd11b-Dtr or wild-type mice administered biologically inactive [Glu ${ }^{52}$ ] DT or PBS showed normal implantations on day 7.5 (Figure 2, C and E). These results indicate that depletion of $\mathrm{CD} 11 \mathrm{~b}^{+}$cells from mice at any stage of the pre-implantation period of early pregnancy leads to complete infertility.

Neutrophils and some dendritic cell lineages can express CD11b. FACS analysis of uterus and ovary tissue from DT-treated Cd11bDtr mice showed that in addition to macrophage loss, there was a decline in $\mathrm{CD}_{11 \mathrm{c}^{+}}$dendritic cells and $\mathrm{RB}^{+}$neutrophils, although these leukocytes comprise smaller populations and their depletion was less extensive than for $\mathrm{F} 4 / 80^{+}$macrophages (Supplemental Table 1). To investigate the leukocyte lineage responsible for infertility, DT-resistant wild-type bone marrow-derived monocyte/ macrophages $\left(\mathrm{F} 4 / 80^{+} \mathrm{CD} 11 \mathrm{~b}^{+}\right.$cells, Supplemental Figure 2$)$ were administered i.v. to Cd11b-Dtr mice on both day $0.5 \mathrm{pc}$ and day 3.5 $\mathrm{pc}$, prior to DT injection on day $3.5 \mathrm{pc}$. After macrophage replacement, implantation sites were evident in 64\% (7/11) Cd11b-Dtr mice at day $7.5 \mathrm{pc}$ (Figure 2, B and E). This demonstrates that the infertility caused by depletion of $\mathrm{CD} 11 \mathrm{~b}^{+}$cells can be attributed to macrophage deficiency.

Infertility after macrophage depletion results from embryo implantation failure. To determine whether macrophage depletion causes loss of implantation sites due to altered embryo development, wild-type and Cd11b-Dtr mice were given DT on day $2.5 \mathrm{pc}$, and embryos were 
A
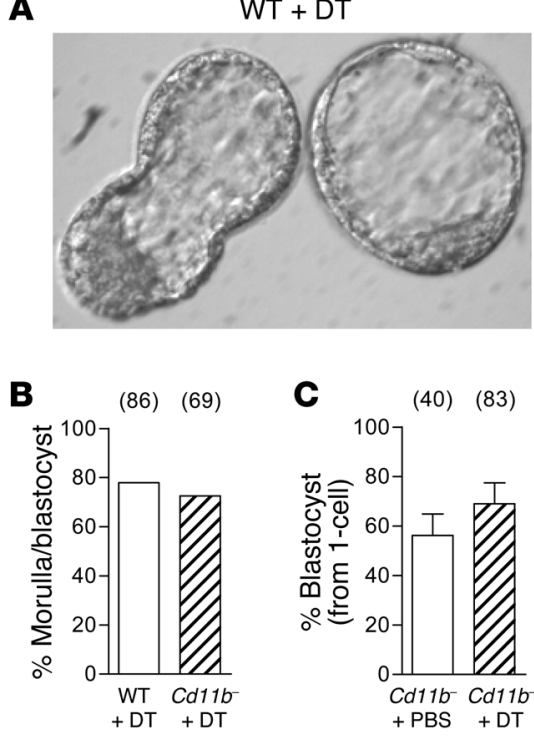

C

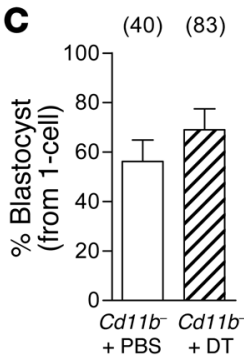

$C d 11 b^{-}+\mathrm{DT}$

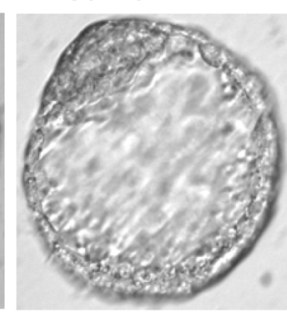

D

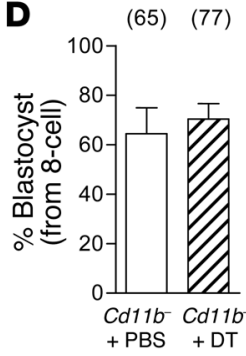

\section{Figure 3}

Implantation failure in macrophage-depleted Cd11b-Dtr mice is not due to adverse effects on pre-implantation embryo development. (A) Embryos flushed on the morning of day 3.5 pc from macrophagedepleted Cd11b-Dtr mice, 24 hours following i.p. injection of DT (25 $\mathrm{ng} / \mathrm{g}$ ), were generally developed to blastocyst stage and often hatched from the zona pellucida, comparable to those flushed from wild-type mice treated with DT. (B) A comparable proportion of embryos flushed from the uterus on day $3.5 \mathrm{pc}$ were developed to morula or blastocyst stage in wild-type or Cd11b-Dtr mice administered DT on day $2.5 \mathrm{pc}$. (C) Embryos that were flushed from the oviduct on day $0.5 \mathrm{pc}$ (1-cell embryos) of gonadatropin-primed Cd11b-Dtr mice and cultured in the presence of DT $(25 \mathrm{ng} / \mathrm{ml})$ for 48 hours, and then without DT for a further 48 hours, developed to blastocyst stage at the same rate as embryos cultured without DT. (D) Embryos flushed from the uterus on day $2.5 \mathrm{pc}$ (8-cell embryos) and cultured with DT $(25 \mathrm{ng} / \mathrm{ml})$ for 72 hours developed to blastocyst stage at the same rate as embryos cultured without DT. (B-D) The number of embryos in each group is shown in parentheses. Data are mean \pm SEM.

implantations were evident, with blastocyst stage embryos lodged in crypts at the anti-mesometrial pole of a narrowed endometrial lumen. Epithelial cells were displaced subjacent to the embryo, with a clear zone of decidual transformation in the uterine stroma cells (Figure 4C). In Cd11b-Dtr females, the lumen often lacked the slit-like narrowed architecture, and the majority of blastocysts were attached laterally or in the middle of an open lumen with no decidual transformation, although decidual transformation with incomplete uterine closure was occasionally seen (Figure 4C). These observations support the interpretation that when macrophages are depleted from Cd11b-Dtr mice, embryo implantation rarely proceeds beyond the initial attachment phase, due to an impaired uterine response associated with disrupted blastocyst apposition, uterine closure, and decidual transformation.

Macrophage depletion disrupts corpus luteum structure and impairs progesterone synthesis. A key determinant of adequate endometrial receptivity and the decidual response to embryo implantation is synthesis of progesterone by the corpora lutea within the ovaries. To investigate the effect of macrophage depletion on luteal function, ovaries were recovered from wild-type and Cd11b-Dtr mice 24 hours following injection with DT on day 3.5 pc. Ovaries from macrophage-depleted Cd11b-Dtr mice were hemorrhagic in appearance, and histology showed a highly irregular architecture within most corpora lutea, consistent with extensive structural disruption (Figure 5A), while the number and distribution of developing follicles appeared normal (Supplemental Table 2). In high-power image sections, the density of luteal cells in Cd11bDtr mice given DT was substantially less than that in wild-type mice, and instead interstitial spaces filled with red blood cells were present throughout the corpora lutea.

Given the clear demise of corpora lutea integrity following macrophage depletion, genes known to be involved in functional and structural luteolysis during normal involution $(29,30)$ were investigated. Prostaglandin-endoperoxide synthase 2 (Ptgs2), which encodes PTGS2 (also known as COX2), a key enzyme in ovarian synthesis of the luteolytic prostaglandin PGF2A, was upregulated more than 1,000-fold in Cd11b-Dtr mice compared with wild-type mice at day 4.5 pc, 24 hours following DT treatment. Induction of 
A
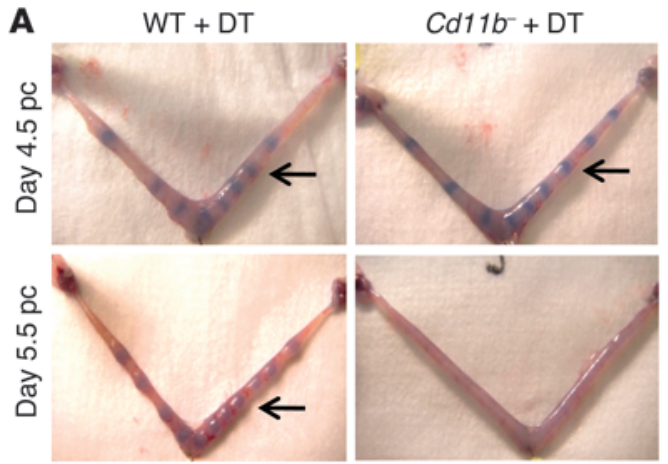

C
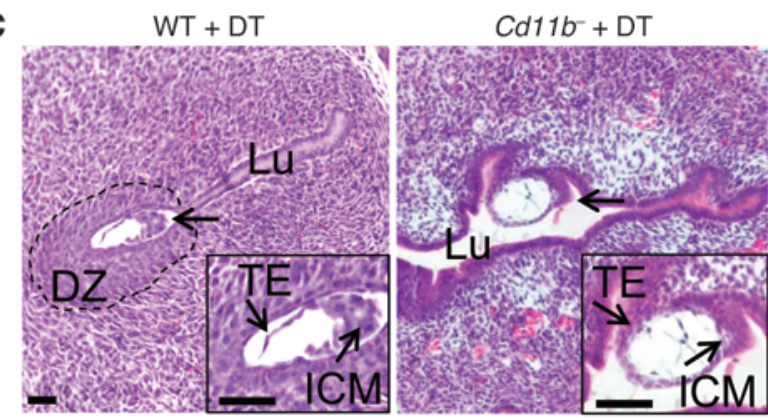

B
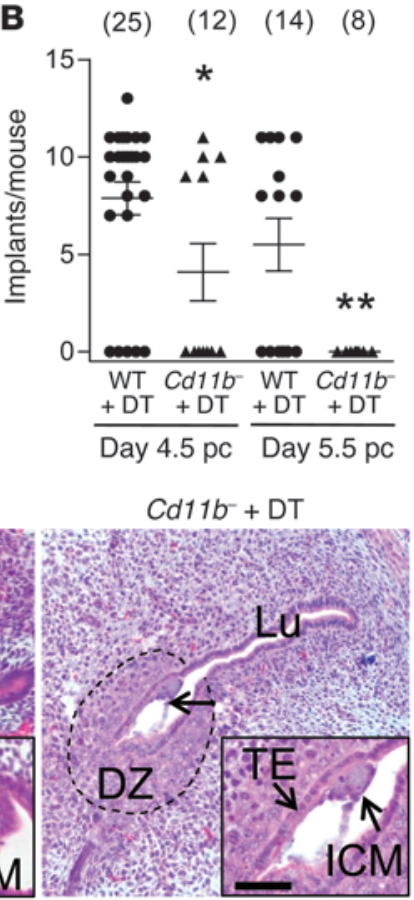

Figure 4

Infertility in macrophage-depleted Cd11b-Dtr mice results from implantation failure. (A) Trypan blue clearly delineates bands of increased vascular permeability in the uterus, showing embryo implantation sites in some, but not all, macrophage-depleted Cd11b-Dtr mice (arrows) on day $4.5 \mathrm{pc}$ (upper right), 24 hours after i.p. injection with DT (25 ng/g) on day $3.5 \mathrm{pc}$, compared with the majority of wild-type mice given DT (upper left). By day 5.5 pc, 48 hours after DT injection, no evidence of implantation was observed in any Cd11b-Dtr mice compared with normal implantation sites in wild-type mice. (B) The numbers of implantation sites per mouse at day $4.5 \mathrm{pc}$ and day 5.5 pc are shown for wild-type mice (WT +DT) and macrophage-depleted Cd11b-Dtr mice (Cd11b+ DT), after injection of DT on day $3.5 \mathrm{pc}$. Data are number of implantations per mouse, with mean \pm SEM superimposed. The number of mice per group is shown in parentheses. ${ }^{*} P<0.05$, ${ }^{\star *} P<0.01$, Cd11b- +DT versus WT +DT. (C) Sections of uterus (H\&E) from Cd11b-Dtr mice on day $4.5 \mathrm{pc}$ (24 hours following DT injection) show that blastocyst-stage embryos (arrows) were attached laterally or in the middle of an open lumen with no decidual zone (10/12 embryos; middle panel), or less frequently were attached with a decidual zone, but incomplete uterine closure (2/12 embryos; right panel). This compared with wild-type mice, in which typical implantation sites in a narrowed endometrial lumen, with surrounding decidual zone, were consistently evident (left panel). Images are representative of 10-12 embryos in 4 WT and 5 Cd11b-Dtr mice. Insets are high-power. Lu, lumen; ICM, inner cell mass; TE, trophectoderm; DZ, decidual zone. Scale bars: $50 \mu \mathrm{m}$.

Ptgs2 also accompanied the infertility that resulted from macrophage depletion at earlier pre-implantation time points, with similar upregulation evident in ovaries collected from Cd11b-Dtr mice on day 1.5 pc or day 2.5 pc (both 24 hours after DT treatment), when the corpus luteum is rapidly developing (Figure 5B). Consistent with disrupted blood flow into the corpus luteum, expression of Hif1a, which functions as the master regulator of the cellular and systemic response to hypoxia, was elevated approximately 2 -fold at each of the pre-implantation time points (Figure 5C). Key inflammation regulators generally suppressed in pregnancy and known to be detrimental to corpus luteum function $(31,32)$ were also induced following macrophage depletion, notably Il6, which was elevated more than 2,000-fold on day 4.5 pc following macrophage depletion and to a comparable extent at earlier time points (Figure 5D). Tnfa was elevated 3-fold on day $4.5 \mathrm{pc}$ and up to 4-fold at earlier time points (Figure $5 \mathrm{E}$ ). Genes regulating apoptosis were also assessed in tissue collected at day $4.5 \mathrm{pc}$. There was a 2.2-fold increase in Hsp90aal gene and a trend to elevated Bax expression, but Bcl2 and Hsp90aa2 were unchanged (Figure 5F).

Given the disruption to corpora lutea and the significant upregulation in proinflammatory genes in the ovary, we next determined the effect of macrophage depletion on ovarian progesterone production in Cd11b-Dtr mice. In plasma collected 8 hours after DT treatment on day $3.5 \mathrm{pc}$, progesterone concentration was not changed, but at 24 hours following DT treatment, plasma progesterone was reduced by $78 \%$ in Cd11b-Dtr mice compared with wild-type controls (Figure $6 \mathrm{~A})$. In contrast, there was no significant effect on circulating estradiol in Cd11bDtr mice (Figure 6A). A similar decline in progesterone was seen if macrophages were depleted 24 hours earlier, on day $2.5 \mathrm{pc}$ (data not shown). To investigate whether disruption to pituitary regulation of the ovary might be responsible for corpus luteum demise, we also measured plasma prolactin (PRL). There was no significant difference in plasma PRL between Cd11b-Dtr and wild-type mice 24 hours (Figure $6 \mathrm{~A}$ ) or at 48 hours after DT treatment (Supplemental Figure 4), indicating that any effect of macrophage depletion on hypothalamic-pituitary axis function was unlikely to be causal in the infertility defect.

To further examine steroidogenic function in corpora lutea after macrophage depletion, genes encoding enzymes involved in progesterone synthesis were measured. Moderate reductions in expression of steroid acute regulatory protein (Star) (Figure 6B), cytochrome P450, family 11, subfamily a, polypeptide 1 (Cyp11a1) (Figure 6C), and hydroxyl$\delta 5$-steroid dehydrogenase, $2 \beta$ - and steroid $\delta$-isomerase $1(H s d 3 b 1)$ (Figure 6D) were seen in Cd11b-Dtr mice compared with wild-type mice to varying extents at different pre-implantation time points, with consistent reduction in all 3 genes on day $2.5 \mathrm{pc}, 24$ hours after DT treatment on day $1.5 \mathrm{pc}$. Expression of aldo-keto reductase family 1, member C18 (Akr1c18), which encodes $20 \alpha-H S D$, an enzyme induced during physiological regression involved in the breakdown of progesterone (33), was surprisingly also reduced on day $2.5 \mathrm{pc}$ but unchanged at other time points (Figure 6E). There were no significant changes in the expression of luteinizing hormone $(\mathrm{LH})$ receptor $(\mathrm{Lhr})$ or PRL receptor (Prlr) at any time point (Figure 6, F and G, respectively), suggesting no impairment of the capacity for pituitary hormone responsiveness in ovarian cells.

To discount the possibility that DT exerts direct effects on steroidogenic function in luteal cells, DT was added to cultures of luteal cells from ovaries of Cd11b-Dtr mice. No adverse effect of high doses of DT $(1 \mu \mathrm{g} / \mathrm{ml})$ on progesterone secretion was seen 
A

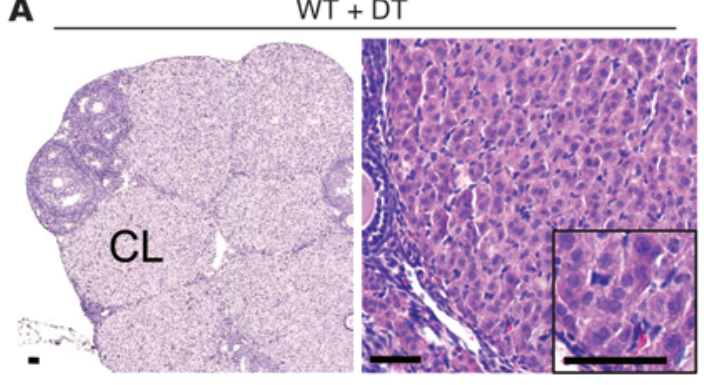

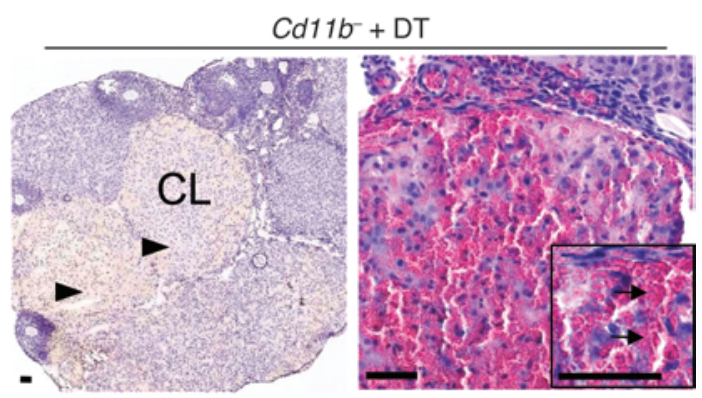

C

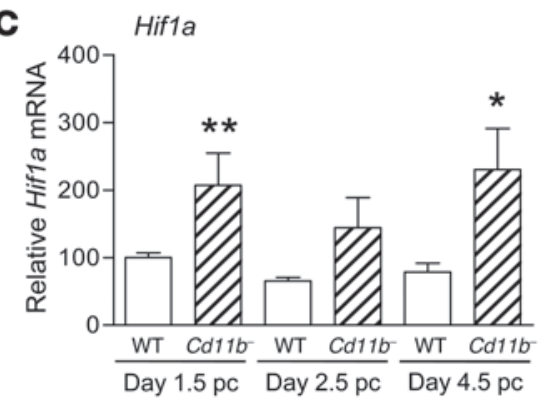

E

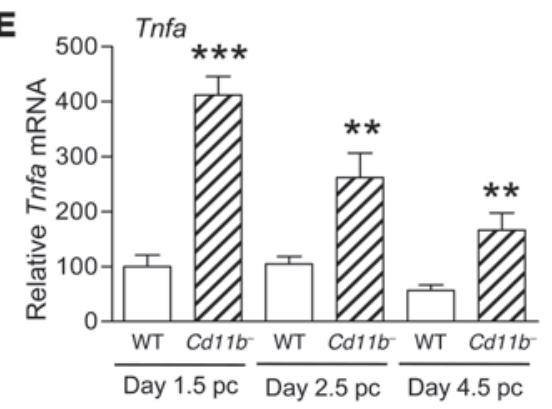

Hif1a
$\mathbf{F}$

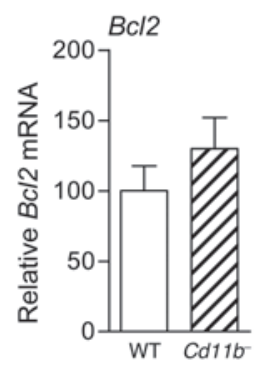

B

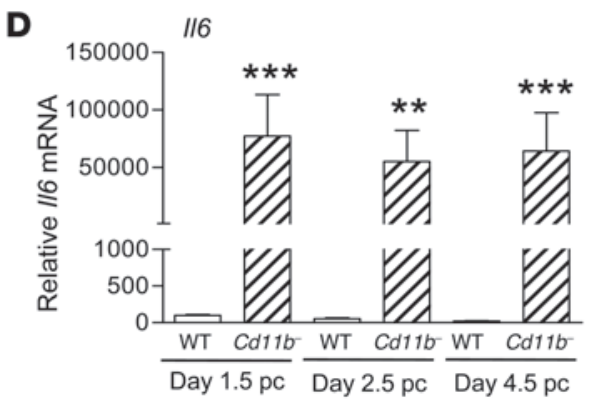

Ptgs2 (Cox2)

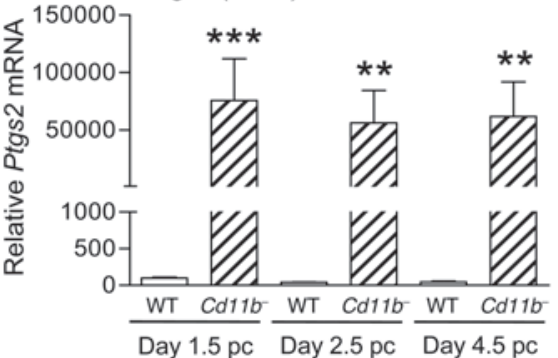

$\frac{\text { WT Cd11b- WT Cd116 }}{\text { Day } 1.5 \mathrm{pc}} \frac{\text { WT Cd11b }}{\text { Day } 2.5 \mathrm{pc}}$

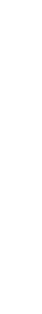


A

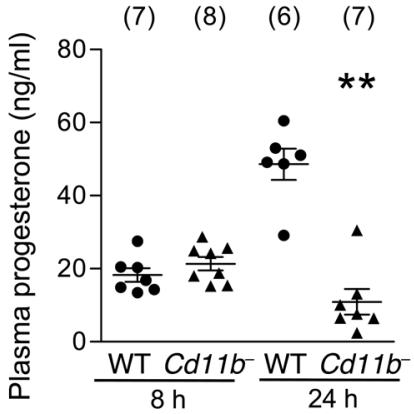

B

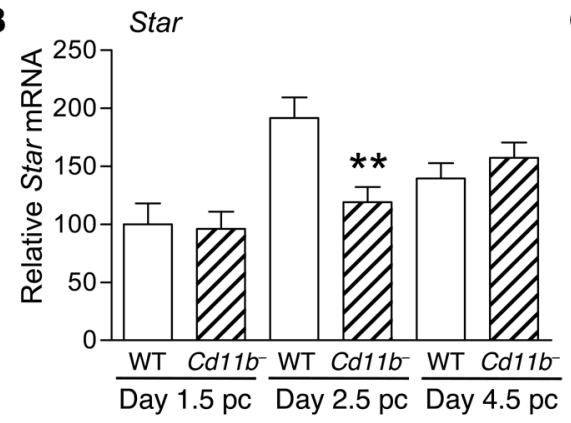

D

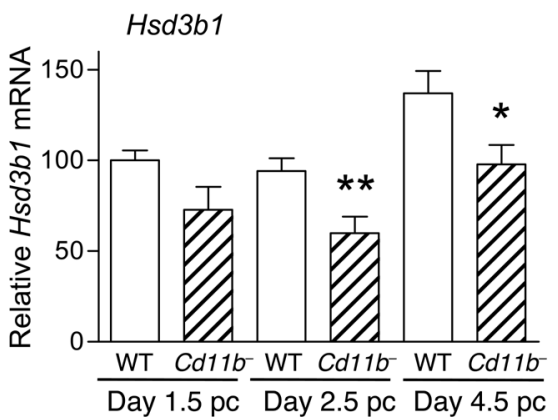

$\mathbf{F}$

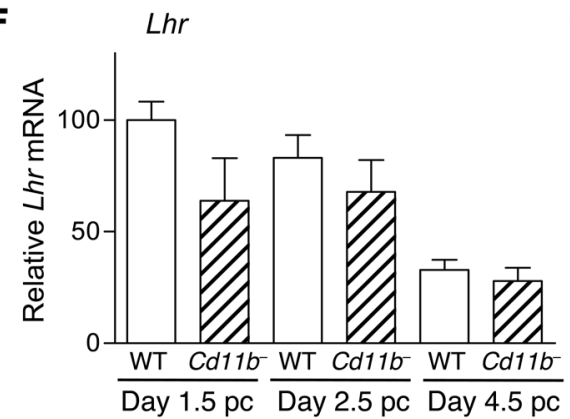

(6)
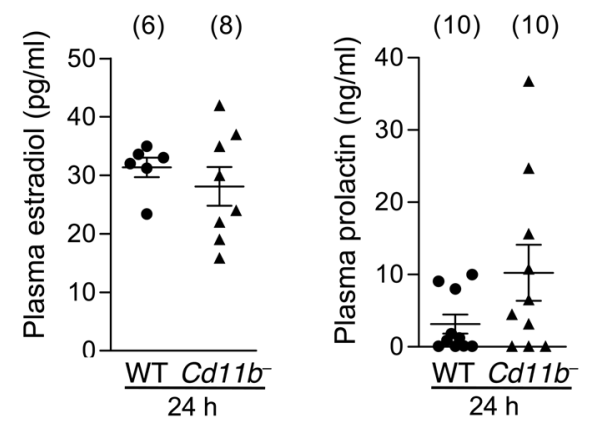

C

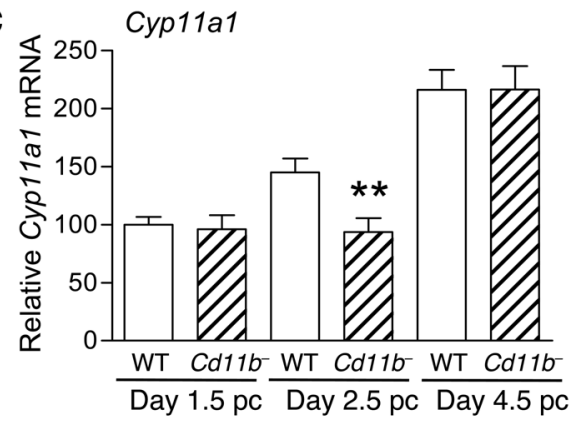

E

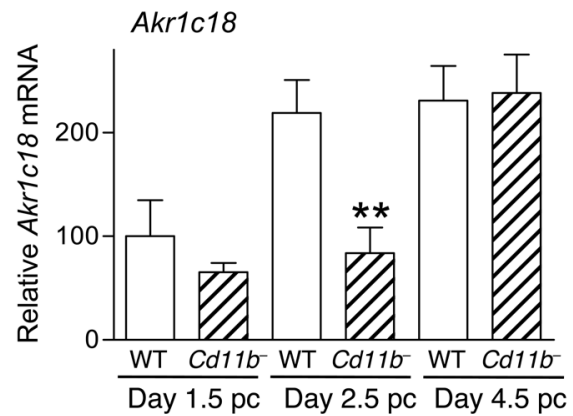

G

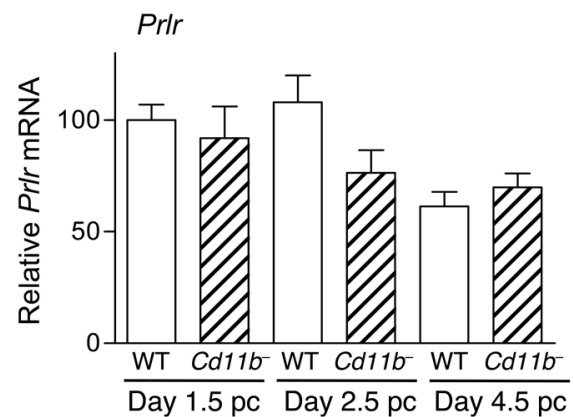

\section{Figure 6}

Luteal defects in macrophage-depleted Cd11b-Dtr mice are associated with diminished plasma progesterone and steroidogenic function. (A) Plasma progesterone in macrophage-depleted Cd11b-Dtr mice was unchanged at 8 hours following i.p. injection of DT (25 $\mathrm{ng} / \mathrm{g}$ ) on day $3.5 \mathrm{pc}$, but by 24 hours was substantially lower than in wild-type mice given DT, while plasma estradiol and PRL were unchanged at 24 hours. Plasma $P R L$ remained unchanged at 48 hours (Supplemental Figure 4). (B-G) Expression of genes regulating steroidogenesis, including (B) Star, (C) Cyp11a, (D) Hsd3b1, and (E) Akr1c18, was downregulated in ovaries of $C d 11 b$ Dtr mice on day $2.5 \mathrm{pc}$ and day $4.5 \mathrm{pc}$, 24 hours after DT injection, compared with wild-type mice given DT. Expression of genes encoding (F) Lhr and (G) Prlr was unchanged on days $1.5,2.5$ and 4.5 pc following DT injection. All genes were quantified by RT-PCR and normalized to Actb expression ( $n=7-8$ mice per group). Data are mean \pm SEM, relative to wild-type expression at day 1.5. ${ }^{\star} P<0.05,{ }^{*} P<0.01$, Cd11b- +DT versus WT +DT.
Infertility after macrophage depletion is rescued by exogenous progesterone administration. We next investigated whether disrupting a role for uterine macrophages in facilitating the decidual response might explain the infertility defect. The effect of macrophage depletion on deciduoma formation was examined in ovariectomized Cd11b-Dtr mice, in whom exogenous progesterone and estrogen were administered systemically to replicate the physiological levels of early pregnancy, before instillation of sesame oil into one horn of the uterus. The decidual response to the oil stimulus occurred in Cd11b-Dtr mice regardless of treatment with DT to remove macrophages or PBS control (Figure 7A), with a similar relative weight gain due to deciduoma in the uterine horn with oil (Figure 7B) and a similar area of deciduoma tissue in uterine sections stained with alkaline phosphatase (Figure 7C). This result indicates that macrophage depletion does not directly impair the uterine decidual response to embryo implantation and indicates an upstream defect.

Next we investigated whether insufficient progesterone might underpin implantation failure, since the uterine decidual response and other changes triggered by the implanting embryo are progesterone dependent. To directly evaluate the effect of replacing luteal progesterone, DT was injected on day $3.5 \mathrm{pc}$ into wild-type and Cd11b-Dtr mice, and the mice were then administered progester- 
A
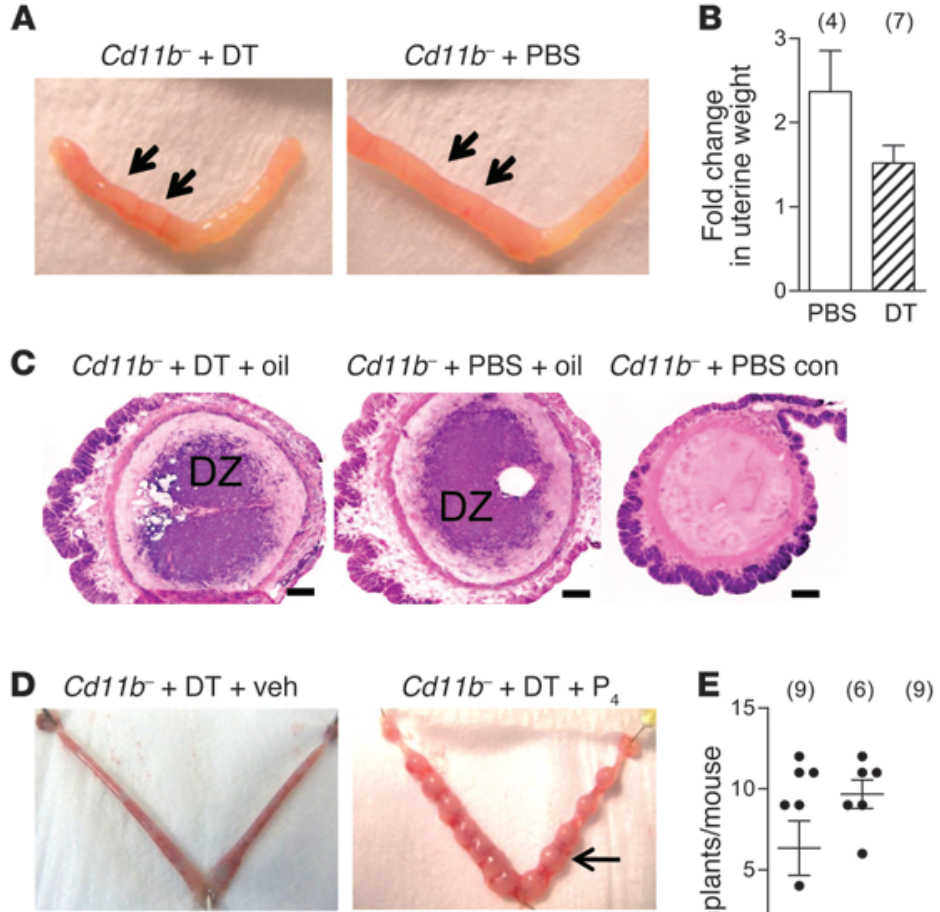

\section{Figure 7}

Infertility in macrophage-depleted Cd11b-Dtr mice is rescued by administration of exogenous progesterone. (A) Deciduoma formation was unchanged in Cd11b-Dtr mice given DT to deplete macrophages, compared with PBS-treated control Cd11b-Dtr mice. Deciduoma formation was measured after oil instillation into the left uterine horn (arrows), following ovariectomy and exogenous progesterone and estrogen replacement to mimic the physiological hormone environment of early pregnancy. (B) The fold-change in weight due to deciduoma (weight of oil-treated uterine horn / weight of control uterine horn) was comparable in Cd11b-Dtr mice given DT or PBS. (C) Alkaline phosphatase staining to detect decidual cells in sections of uterus from the oil-instilled left horn of the uterus showed a similar area of deciduoma in DT-treated and PBS-treated Cd11b-Dtr mice, with no deciduoma in the right, control horn. Scale bars: $200 \mu \mathrm{m}$. (D) At autopsy on day 7.5 pc, macrophage-depleted Cd11bDtr mice had normal implantation sites (arrow) when progesterone $\left(\mathrm{P}_{4}\right)$ was administered following injection of DT $(25 \mathrm{ng} / \mathrm{g})$ on day $3.5 \mathrm{pc}$, compared with absence of implantation sites in macrophage-depleted Cd11b-Dtr mice given vehicle (veh; left panel). (E) The number of implantation sites per mouse is shown for wild-type mice (WT +DT) and macrophage-depleted Cd11b-Dtr mice (Cd11b- +DT) administered DT on day $3.5 \mathrm{pc}$, followed by progesterone or vehicle. Data represent the number of implantations per mouse, with mean \pm SEM superimposed. The number of mice in each group is shown in parentheses. ${ }^{*} P<0.0001, C d 11 b-+D T$ versus WT +DT. one or vehicle daily from days 3.5 to 6.5 pc. Eleven of twelve (92\%) macrophage-depleted, progesterone-administered Cd11b-Dtr mice carried viable pregnancies when autopsied on day $7.5 \mathrm{pc}$, while there were no viable pregnancies (0/9) in macrophage-depleted Cd11b-Dtr mice treated with vehicle (Figure 7, D and E). Furthermore, implantation sites were similar in size (data not shown) and comparable in number to those in DT-treated wild-type mice administered vehicle or progesterone (Figure 7E).

To determine whether exogenous progesterone could alleviate infertility induced by macrophage depletion at an earlier preimplantation time point, an additional group of Cd11b-Dtr mice were given DT on day $0.5 \mathrm{pc}$ and then daily progesterone injections until day 6.5 pc. On day 7.5 pc, 71\% (5/7) carried viable pregnancies, and the number of implantation sites was similar to that of wild-type controls (mean \pm SEM, $6.1 \pm 1.8$ per mouse). These results clearly indicate that the disrupted decidual response and subsequent implantation failure in macrophage-deficient $C d 11 b$ $D t r$ mice is a direct consequence of progesterone deficiency. Normally developed fetuses were present in a proportion of $C d 11 b-D t r$ females treated with DT at day $3.5 \mathrm{pc}$ and then administered progesterone daily until late gestation (day 17.5 pc) (Supplemental Table 3). This indicates that if progesterone insufficiency is alleviated, normal progression of pregnancy is possible despite transient macrophage depletion in the peri-implantation period.

Corpus luteum macrophages have a pro-angiogenic phenotype and their depletion impairs development of the microvascular network. To investigate the mechanism by which macrophages affect corpus luteum structure and function, the effect of macrophage depletion on the microvascular endothelial cell network was examined using the endothelial cell antibodies MTS-12 and CD31 (PECAM1). In untreated wild-type and in CD11b-Dtr mice, an intricate network of blood vessels within the corpus luteum and ovarian stroma was observed on day $3.5 \mathrm{pc}$ (Figure 8A). However, following DT-elicited macrophage depletion in Cd11b-Dtr mice, endothelial cells were substantially diminished in number and the majority of corpora lutea showed a complete absence of the vascular network, or patchy distribution of vessels with evidence of disturbed arborization (Figure 8B). An intimate juxtaposition between macrophages and endothelial cells was evident when ovarian tissue sections were double-labeled for endothelial cell marker CD31 and macrophage marker F4/80 (Figure 8C). Interestingly, a small number of cells co-expressing both CD31 and F4/80 were observed (Figure 8D). In contrast, the blood vessels within the ovarian stroma surrounding the corpora lutea remained intact (Figure $8 \mathrm{~B}$ ) and the lymphatic vessels in stromal tissue were unaffected (Figure 8E) after macrophage depletion in Cd11b-Dtr mice.

The close physical association between macrophages and endothelial cells suggests that macrophages may provide trophic support for endothelial cells during neovascularization in the nascent corpus luteum. In developing fetuses and in tumors, macrophages with a proangiogenic phenotype can be identified on the basis of their expression of the tyrosine kinase receptor TIE2 (CD202b) (34). Consistent with a proangiogenic phenotype, we found TIE2 was expressed by corpus luteum macrophages at day 2.5 pc (Figure 9A, top). This contrasted with macrophages in the ovarian stroma, where TIE2 was only rarely expressed (Figure 9A, bottom). Within the corpus luteum, F4/80+ macrophages expressed TIE2 at an intensity comparable to the weak expression seen on neighboring blood vessel endothelial cells, which contrasted with the strong expression seen on mature blood vessels in the ovarian stroma (Supplemental Figure 6). 
A
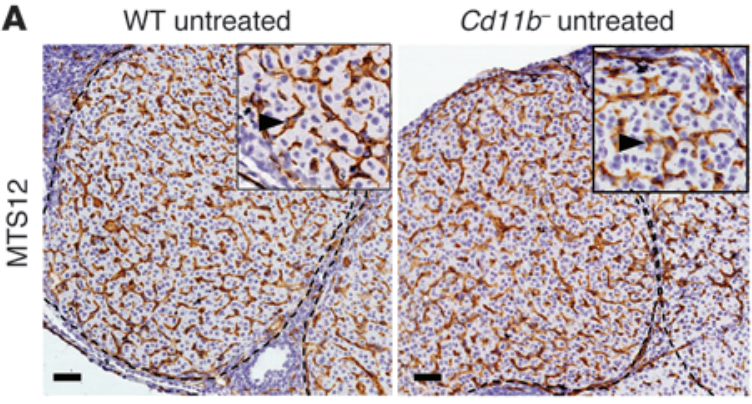

B

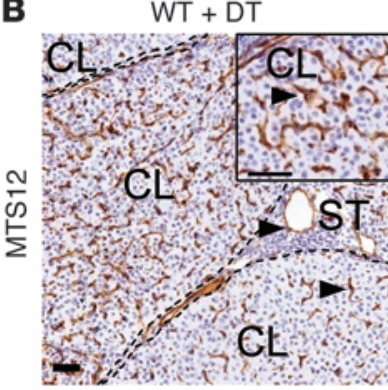

C

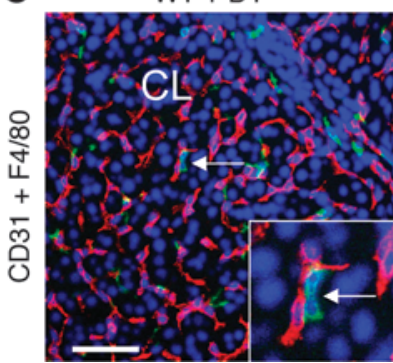

Cd11b- DT

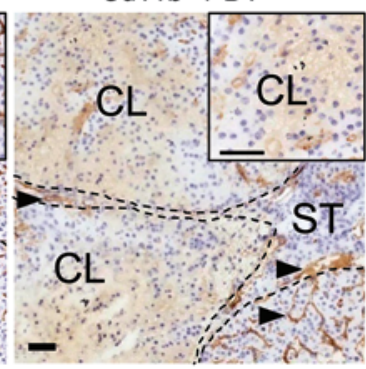

Cd11b- + DT

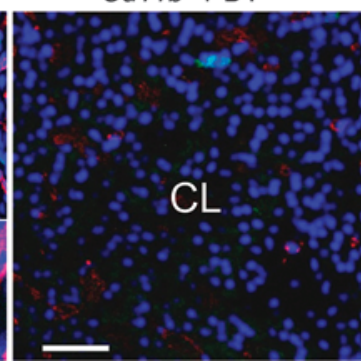

D
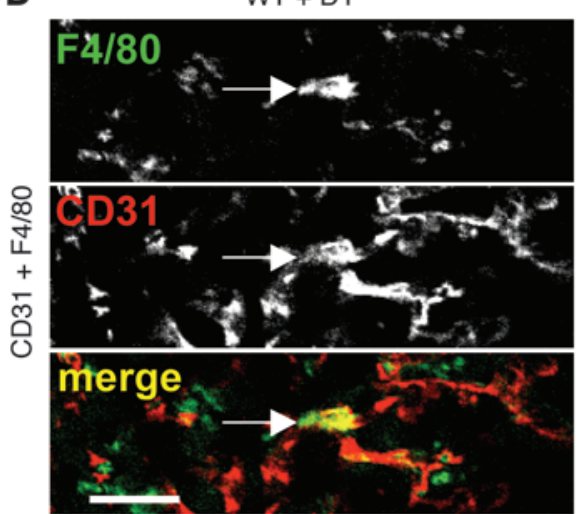

E
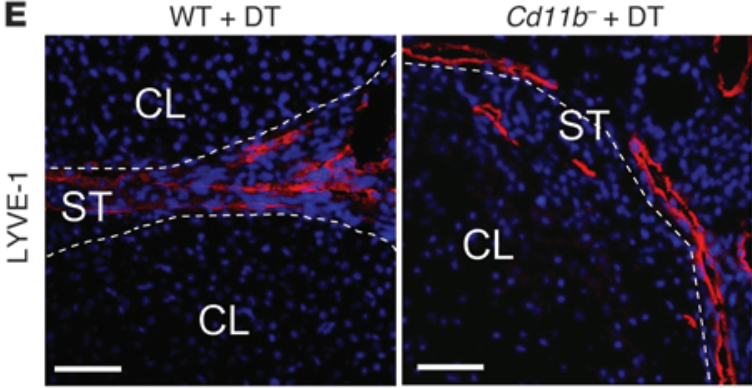

\section{Figure 8}

The luteal defect in macrophage-depleted Cd11b-Dtr mice is accompanied by breakdown of the corpus luteum vasculature. (A-E) Ovaries were recovered from wild-type control or Cd11b-Dtr mice on day 3.5 pc before treatment (A) or on day 4.5 pc, 24 hours after i.p. injection of DT (25 $\mathrm{ng} / \mathrm{g}$ ) (B-E). (A) Sections labeled with MTS-12 to detect blood vessel endothelial cells indicated a substantial network of intact vessels (arrows) in the corpus luteum of both Cd11b-Dtr and wild-type mice on day 3.5 pc. (B) Sections labeled with MTS-12 to detect blood vessel endothelial cells indicated few intact vessels in the corpus luteum of most Cd11b-Dtr compared with wild-type mice, while vessels in the ovarian stroma (arrowheads) and some corpora lutea (arrows) remained intact on day 4.5 pc, following DT injection 24 hours earlier. (C) Sections labeled with antibodies to both CD31 (red) to detect endothelial cells and F4/80 (green) to detect macrophages showed absence of blood vessels in the corpus luteum of $C d 11 b$-Dtr compared with wild-type mice, highlighting the close spatial association between endothelial cells and macrophages (inset is high power). (D) In sections labeled with both CD31 and F4/80, cells co-expressing both markers (arrows) were evident. (E) Sections labeled with LYVE-1 (red) to detect lymphatic endothelial cells showed lymphatic vessels at the margins of a corpus luteum of Cd11b-Dtr mice, similar to wild-type mice. Photomicrographs are representative of 6-7 mice per group. Scale bars: $50 \mu \mathrm{m}$.

To further investigate the role of macrophages in corpus luteum angiogenesis, expression of genes encoding VEGFs that regulate angiogenesis were quantified in ovaries from wild-type and Cd11b-Dtr mice 24 hours following DT administration at various time points during the pre-implantation period. A marked upregulation of Vegfa mRNA was evident following macrophage depletion (>3.4-fold compared with wild-type mice) (Figure 9B), as is commonly observed in hypoxic conditions secondary to Hifla expression (35). No effect on the expression of Vegfb mRNA was observed (Figure 9C), but on days $1.5 \mathrm{pc}$ and $2.5 \mathrm{pc}$, expression of Vegfc mRNA in DT-treated Cd11b-Dtr mice was decreased $40 \%$ compared with control mice (Figure 9D), and on day $1.5 \mathrm{pc}$, expression of Figf mRNA was decreased by $30 \%$ (Figure 9E). This diminished synthesis of VEGFC and FIGF (VEGFD) following macrophage depletion is consistent with macrophage production of these VEGFs to support luteal angiogenesis in the periimplantation period.

\section{Discussion}

In this study the conditional macrophage depletion model afforded by $C d 11 b$-Dtr mice was used to deplete macrophages from the uterus and ovary, allowing for identification of the key roles of macrophages in the event of embryo implantation. Our experiments provide evidence that macrophages have an essential role in establishing pregnancy in mice, through support of the development and structural integrity of the corpus luteum vasculature essential for normal steroidogenic function. We show that removal of macrophages during early pregnancy results in 

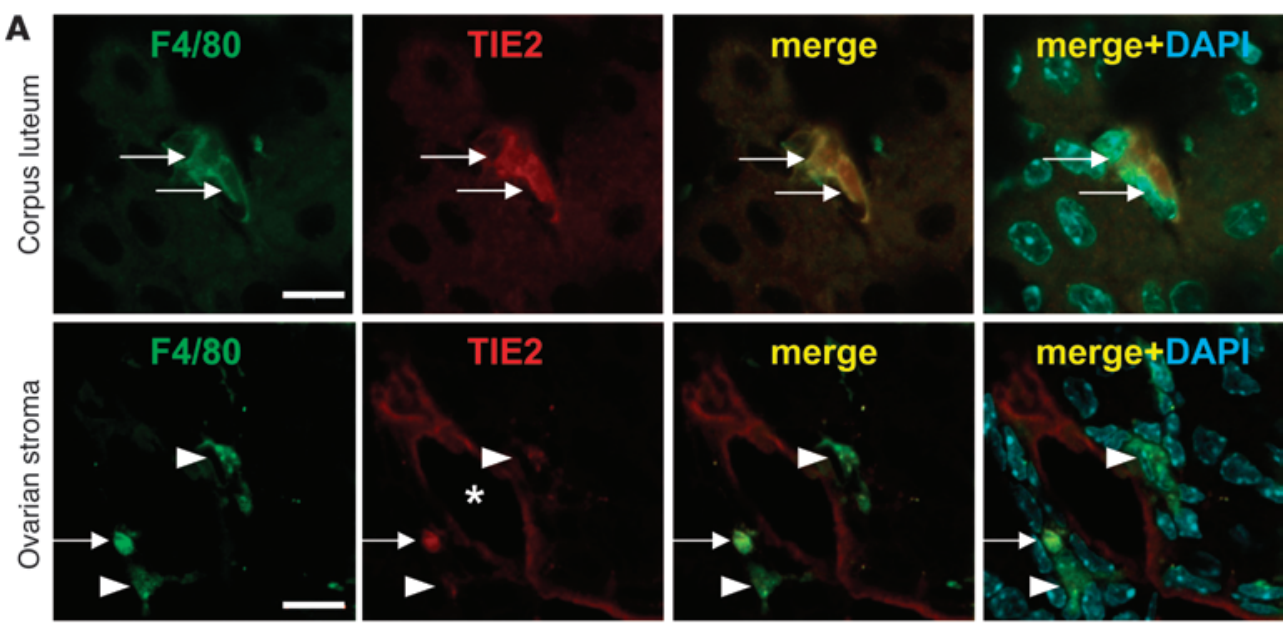

B

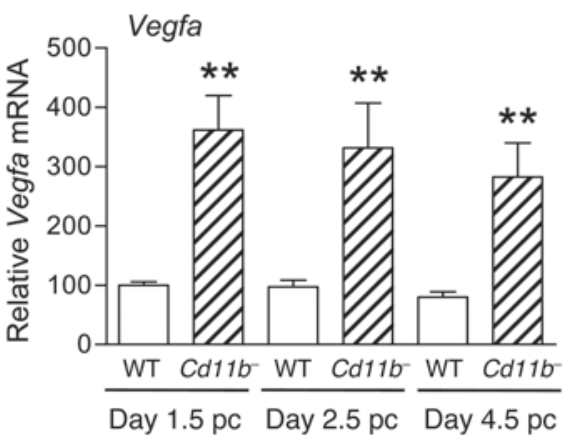

C

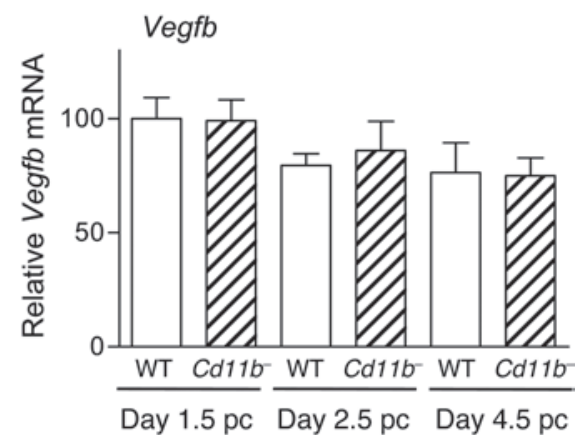

D

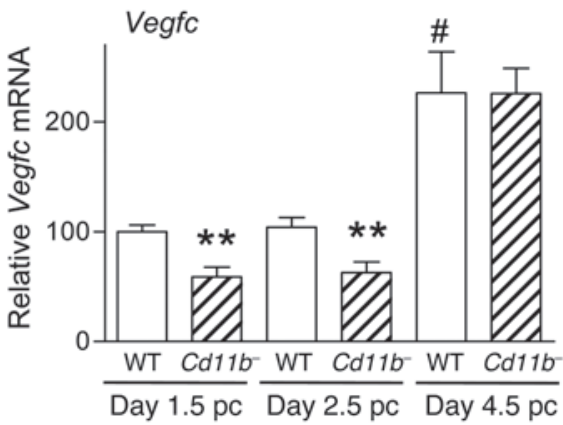

E

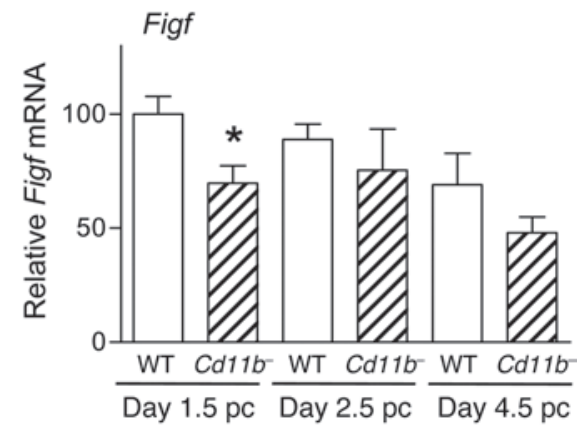

\section{Figure 9}

Macrophages in the corpus luteum have a proangiogenic phenotype, and macrophage depletion in Cd11b-Dtr mice is accompanied by altered expression of VEGF genes. (A) Sections of ovary from wild-type mice on day 2.5 pc labeled with antibodies to TIE2 and F4/80 showed that macrophages in the corpus luteum were $\mathrm{TIE2}^{+}$, indicative of a proangiogenic phenotype. Both $\mathrm{TIE2}^{+}$and $\mathrm{TIE}^{-}$macrophages were seen adjacent to TIE2+ blood vessels in the ovarian stroma (arrows, F4/80+TIE2+ macrophages; short arrows, F4/80+TIE2- macrophages; *vessel lumen). Scale bars: $10 \mu \mathrm{m}$. (B-E) Expression of angiogenesis genes was altered in ovaries in association with macrophage depletion from Cd11b-Dtr mice. (B) Vegfa was upregulated in ovaries of Cd11b-Dtr mice on days 1.5 pc, 2.5 pc, and 4.5 pc, 24 hours following DT injection, compared with wild-type mice given DT. (C) Vegfb was not changed at any time point following DT injection. (D) Vegfc was downregulated in ovaries of Cd11b-Dtr mice on days 1.5 and 2.5 pc following DT injection. (E) Figf was downregulated in ovaries of Cd11b-Dtr mice on day 1.5 pc following DT injection. All genes were quantified using RT-PCR and normalized to Actb expression ( $n=6-10$ mice per group). Data are mean \pm SEM relative to wild-type expression at day $1.5 .{ }^{*} P<0.05,{ }^{*} P<0.01, C d 11 b-+$ DT versus WT + DT at same time point; ${ }^{*} P<0.01$ WT + DT at day 4.5 pc versus day 1.5 pc.

failure of embryo implantation, and this is due to insufficient progesterone production secondary to destruction of the dense capillary network that is essential for sustenance and support of the maturing corpus luteum. This absolute requirement for macrophages provides new insight into corpus luteum biology and reveals macrophages as a potentially critical limiting factor contributing to luteal dysfunction implicated in some forms of infertility. If a similar role is confirmed in women, macrophages may comprise a new target for therapeutic intervention to promote optimal ovarian function.

In the hours after ovulation, the surge in $\mathrm{LH}$ and breakdown of the follicular basement membrane initiates a tightly regulated sequence of molecular events, under control of pituitary PRL and local signaling regulators to form the corpus luteum $(29,30)$. 
Migration of endothelial cells, macrophages, and other leukocytes, fibroblasts, and theca cells into the previously avascular granulosa layer is followed by rapid neovascularization involving endothelial cell proliferation, followed by expansion and maturation of capillaries, to form a dense vascular bed within 24 hours after ovulation (36). Bone marrow-derived endothelial progenitor cells recruited into the follicle prior to ovulation reportedly contribute to the formation of blood vessels in the nascent corpus luteum (37), and our finding of $\mathrm{CD} 31^{+} \mathrm{F} 4 / 80^{+}$cells is consistent with this. Through direct contact with several capillaries, every steroidogenic luteal cell is thereafter provided with the nutrients, hormones, oxygen, and cholesterol substrate to support progesterone synthesis and export to the circulation. The corpus luteum and its supporting vasculature continues to expand over the pre-implantation phase to steadily increase output of progesterone. In turn, progesterone circulates to the uterus to induce the adaptations required for embryo attachment, luminal closure, decidual transformation, and the vascular changes that are required for placental development.

In the current study, macrophage depletion at any time from conception to embryo implantation reliably caused implantation failure. Although small implantation sites and decidual cells were sometimes detectable at day 4.5 pc in macrophage-depleted mice, pregnancy inevitably failed by day $5.5 \mathrm{pc}$ in all mice studied. Embryos were impervious to adverse effects of DT, and embryos flushed in the pre-implantation phase from reproductive tracts of macrophage-depleted females were normally developed. Together these observations imply that without $\mathrm{CD}_{11} \mathrm{~b}^{+}$cells, implantation failure occurs during the initial steps of embryo attachment and the ensuing decidual response.

A limitation of the Cd11b-Dtr model is that cell lineages including neutrophils and dendritic cells are also affected by DT administration, likely due to both $C d 11 b$ promoter-driven expression of the Dtr transgene as well as lineage and trophic relationships with monocyte/macrophages. However, since reconstitution with wildtype $\mathrm{F} 4 / 80^{+} \mathrm{CD} 11 \mathrm{~b}^{+}$cells alleviated the infertility phenotype, we are confident that macrophages are the cells responsible for the observed effects.

The progression of viable pregnancy in macrophage-depleted $C d 11 b$-Dtr mice was consistently rescued when exogenous progesterone was administered daily from the time of macrophage depletion. Progesterone is a critical regulator of the structural and secretory changes that support endometrial receptivity to implantation and early placental morphogenesis (38). Thus the primary cause of pregnancy failure after macrophage depletion can be attributed to diminished ovarian progesterone, without which the uterine endometrium is inadequately prepared for embryo implantation and the decidual response. That exogenous progesterone is sufficient to rescue implantation demonstrates that the disturbances to uterine receptivity in early pregnancy are secondary to the ovarian lesion. Although our findings do not rule out regulatory effects of macrophages on post-implantation events, the results of the progesterone rescue experiment implies that macrophages do not have essential independent actions in the events of conception, embryo attachment, or decidualization and alleviates any concern that off-target effects of DT might contribute to the infertility seen in the Cd11b-Dtr model.

The absolute requirement for macrophages in the developing corpus luteum was directly linked to a role in formation of the vascular network and its structural integrity, since depletion of macrophages from Cd11b-Dtr mice caused a dramatic disintegration of the capillary bed within 24 hours of DT administration. These observations extend the findings of a previous study in which macrophage depletion from non-pregnant, cycling mice resulted in an irregular vasculature in corpora lutea (15). Macrophages can exert potent pro-angiogenic activity through release of a wide range of factors, including VEGF, bFBF, and EGF, that directly activate endothelial cell proliferation and/or induce endothelial cell migration $(2,3)$. Recently a distinct subset of proangiogenic macrophages has been identified, defined by surface expression of the tyrosine kinase receptor TIE2 (39). TIE2-expressing monocytes circulate at low frequency in the peripheral blood and are generally absent from normal adult tissues and organs, but are prevalent in the developing fetus, in some regenerating adult tissues, and in tumors $(34,39)$. TIE2 is expressed abundantly in quiescent endothelial cells of mature blood vessels and at lower levels in angiogenic endothelial cells, where it mediates context-dependent signaling by angiopoietin- 1 and angiopoietin- 2 to control vascular sprouting, vessel assembly, and stabilization (40). This expression pattern is consistent with our observation that corpus luteum vessels expressed less TIE2 than established vessels in the ovarian stroma. Macrophages linked with blood vessel formation have an alternatively activated M2 phenotype $(3,4)$, and their bi-directional communication with endothelial cells supports M2 macrophage differentiation and induces TIE2 expression (41). In this context, it is relevant that macrophages residing in the developing corpus luteum show features of the M2 phenotype, while luteal regression is linked with proinflammatory M1 macrophages (42).

Transgenic studies define VEGFA as a key factor for instructing recruitment of macrophages into sites of neovascularization in adult heart and lung tissue, where they form close associations with endothelial cells and secrete endothelial cell mitogens to amplify the effects of locally produced VEGF and thereby promote vessel formation $(43,44)$. Our results indicate that similar pathways of macrophage-supported vascular development operate in developing corpora lutea. Elevated transcription of genes encoding various VEGFs commences early after ovulation in luteal cells, and VEGF-driven capillary formation is a hallmark of luteinization $(45,46)$. Administration of VEGF trap, a soluble truncated form of the FLT1 receptor, to marmosets after ovulation causes decreased endothelial cell proliferation, failed development of the microvascular tree, and reduced plasma progesterone (47). Even in the mid-luteal phase, when vascularization is largely complete, VEGFA inhibition elicited a rapid decline in progesterone, increased caspase-3-positive endothelial cells, and decreased endothelial cell density (48). In mice, administration of a truncated sFLT1 receptor inhibited angiogenesis and corpus luteum development and decreased progesterone production, while preexisting vasculature elsewhere in the ovary was unaffected (49).

The amplifying properties of macrophages recruited into angiogenic sites are largely mediated by their potent release of VEGFC and FIGF $(44,50,51)$. VEGFC and FIGF bind and activate KDR (VEGF receptor 2) and FLT4 (VEGF receptor 3) to exert mitogenic actions in endothelial cells (52-55). Branching morphogenesis and stabilization of nascent blood vessels in developing vessels appears to be determined by precise patterns of angiogenic signals, with VEGFC release by TIE2 ${ }^{+}$macrophages implicated in controlling specification of endothelial cells (56). Consistent with VEGFC and possibly FIGF production by TIE2 ${ }^{+}$corpus luteum macrophages, we found that Vegfc and Figf expression were diminished in macrophage-depleted Cd11b-Dtr ovaries. Removal of macrophage trophic 
support for microvascular development and maintenance would thus explain the vascular deficit observed after macrophage depletion, and we predict that VEGFC release from TIE2+ macrophages is centrally involved in mediating this role.

The ovarian lymphatics are a major conduit for progesterone efflux from the ovary during the luteal phase (57), and VEGFC and FIGF are also known for promoting lymphangiogenesis (58). However, we found the lymphatic vasculature as well as established blood vessels in the ovarian stroma to be unaltered by macrophage depletion. This indicates that ovarian lymphatics and existing blood vessels are less vulnerable to deprivation of macrophage support than newly formed microvascular structures.

Depletion of macrophages caused an unexpected, marked upregulation of Vegfa mRNA expression. Luteal cells are likely to be the source of this elevated Vegfa expression, potentially in reaction to the insult of acute macrophage demise after DT administration. Hypoxia is a key stimulus for synthesis of angoigenic factors including VEGFA (35), and the observed elevation of Hif1a after macrophage depletion is consistent with a hypoxic environment secondary to destruction of the microvascular network. VEGFA is also a potent inducer of vascular permeability (59), and increased Vegfa expression may contribute to the hemorrhage evident in the ovaries of macrophage-depleted Cd11b-Dtr mice.

Macrophage loss was accompanied by induction of several proinflammatory genes, which is reminiscent of the physiological process of luteolysis $(29,30)$. The corpus luteum is highly sensitive to disruption by innate immune or inflammatory activation leading to pregnancy demise (60). Pro-inflammatory mediators presumably amplify the consequences of withdrawn endothelial cell support, to accelerate vascular destruction and functional and structural demise of the corpus luteum. Most evident was strong induction of Ptgs2, which encodes PTGS2 (Cox2), a key enzyme in ovarian synthesis of the luteolytic prostaglandin $\mathrm{PGF}_{2 \alpha}$, the major luteolytic factor in rodents. Also induced were Il 6 and Tnfa, inflammatory regulators that are detrimental to corpus luteum function and elevated during its structural demise $(31,32)$. TNF has been identified as a key inducer of apoptosis in both luteal cells and endothelial cells in the corpus luteum and would be expected to exacerbate the effects of macrophagederived endothelial mitogen withdrawal (61), while IL-6 production in the corpus luteum is normally silenced for the duration of pregnancy (31). Consistent with macrophage removal causing apoptosis, the ratio of $\mathrm{Bax} / \mathrm{Bcl}$ expression was increased, as occurs in physiological corpus lueum involution $(29,62)$. The finding of elevated heat shock protein is similarly linked with functional demise of progesterone synthesis $(30,63)$.

Similarities between the gene expression changes observed after acute macrophage depletion and those in physiological regression of the corpus luteum raise the question of a possible role for the macrophage-endothelial nexus in executing luteolysis. PGF $2 \alpha$ does not directly alter the hemodynamics of the microvasculature but activates a sequence of events to suppress progesterone production $(30,64)$. After macrophage depletion, the decline in circulating progesterone was associated with reduced expression of the major rate-limiting enzymes involved in progesterone synthesis, Hsd3b1, Cyp11a, and Star. In physiological luteolysis, $\mathrm{PGF}_{2 \alpha}$ diminishes STAR synthesis to impair cholesterol uptake and elicits abrupt upregulation of Akr1c18 encoding 20alpha-HSD, which converts progesterone to pregnenolone. However, macrophage depletion caused inhibition of Akr1c18, which does not reflect the normal events of physiological luteolysis. While the most profound effect of macrophage depletion is exerted on endothelial cells and thus indirectly on luteal cells, loss of direct macrophageluteal cell interaction may also contribute. As reported by other investigators (19), we confirmed in co-culture studies that macrophages can directly enhance luteal cell secretion of progesterone. Additionally the clear demise in patent vessels after macrophage depletion would impair export of progesterone from the ovary.

A key role for an M2 macrophage phenotype in regulating corpus luteum development concurs with previous findings that mice with a genetic deficiency in M-CSF or GM-CSF (CSF2) have diminished progesterone synthesis in early pregnancy $(16,65)$. In Csf1 op/Csf1 op mice, circulating progesterone is $50 \%$ lower than in wild-type mice at implantation, although levels normalize later in pregnancy (16). Ovarian macrophages in Csf2-null mutant mice show elevated nitric oxide production with decreased MHCII and CD11b expression, and progesterone levels are $20 \%$ lower than in wild-type controls (65). TGF- $\beta$ is a paramount regulator of M2 macrophages, and progesterone is reduced by $75 \%$ in Tgfb1-null mutant mice (66).

Obvious vascular disruption has not been reported in studies of Cd11b-Dtr mice that investigated kidney, peritoneum, skin, or mammary gland function $(28,67-69)$. The corpus luteum vasculature is distinguished by its cyclic development and demise; it emerges rapidly over the course of the ovarian cycle and during early pregnancy, before regressing to form the corpus albicans. This contrasts with the mature and long-lived vasculature in nonreproductive organs. By virtue of its transitory appearance and rapid development in each reproductive cycle, the vasculature of the corpus luteum may be more dependent on proangiogenic macrophages than are less dynamic tissues.

Our observations raise the question of whether ovarian macrophages contribute to insufficient luteal phase progesterone production in some infertile women (70). Progesterone supplementation is routinely provided in assisted reproductive treatments (71) and is viewed as a promising therapy for recurrent miscarriage (72). Endogenous and environmental factors are thought to affect macrophage populations in human peripheral tissues. Resident tissue macrophages display marked heterogeneity and have a diverse range of phenotypes, even among different compartments within the same tissue $(1-3,5)$. Environmental factors associated with infertility, including infection, obesity, and stress, all induce inflammatory cytokines and affect macrophage phenotype and function and could well influence macrophage competence to support corpus luteum development (73-75).

In conclusion, our studies reveal that macrophages are required for normal progesterone synthesis in early pregnancy, and their deficiency is incompatible with pregnancy success. The paramount function of macrophages at this time is to provide trophic support for formation of the vascular network, which is critical to corpus luteum function. Collectively, the current work and results of previous studies indicate that fine control of macrophage populations, through regulatory cytokines, chemokines, and other intra-ovarian and systemic factors, would exert a major influence on corpus luteum formation and capacity to support pregnancy. Perturbation of macrophage-endothelial cell crosstalk within the ovary during the peri-conceptional period is likely to be pivotal in luteal insufficiency in women, and there may be therapeutic opportunities to stimulate luteal function through the targeting of macrophages. 


\section{Methods}

Mice and DT treatment. All mice were aged 7-12 weeks at the onset of experiments, were housed under specific pathogen-free conditions at the University of Adelaide Medical School Animal House on a 12-hour light/12hour dark cycle, and were administered food and water ad libitum. Mating was confirmed by detection of a vaginal plug and defined as day $0.5 \mathrm{pc}$. Stages of the estrous cycle were determined by cytological evaluation of vaginal smears performed daily at 9:30 am, as described previously (66). Cd11b-Dtr mice expressing the simian DT receptor driven by the $C d 11 b$ promoter on an inbred FVB/N background (official symbol Tg[ITGAM-DTR/ EGFP]34Lan) (26) were provided by Richard Lang (University of Cincinnati, Cincinnati, Ohio, USA).

To induce macrophage depletion, Cd11b-Dtr mice were given DT from Corynebacterium diphtheria (Sigma-Aldrich) $(25 \mathrm{ng} / \mathrm{g}$ i.p.) $(28,67)$. Nontransgenic wild-type FVB/N mice or PBS-treated Cd11b-Dtr mice were used as controls in all experiments. For all DT-treated Cd11b-Dtr mice, PECs were collected at autopsy and analyzed using flow cytometry with anti-CD $11 \mathrm{~b}$ and anti-F4/80 as described below, to ensure adequate macrophage depletion $\left(<0.5 \% \mathrm{CD} 11 \mathrm{~b}^{+} \mathrm{F} 4 / 80^{+}\right.$cells). As previously reported $(28,67)$, this dose of DT induced macrophage depletion that persisted for approximately 48 hours in Cd11b-Dtr mice, but no effect was observed on macrophage abundance in non-transgenic mice. Additional groups of mice received non-biologically active DT homolog [Glu $\left.{ }^{52}\right]-D T(25 \mathrm{ng} / \mathrm{g}$, i.p.; Sigma-Aldrich) in place of DT, or additional treatment with progesterone ( $2 \mathrm{mg}$ in $100 \mu \mathrm{l}$ sesame oil s.c. daily; Sigma-Aldrich) or sesame oil vehicle control (Sigma-Aldrich).

Assessment of embryo implantation sites. To identify implantation sites at 4 pm on day 4.5 and day 5.5 pc, mice were anesthetized using Avertin (2\% tribromoethanol, $15 \mu \mathrm{l} / \mathrm{g}$ i.p.; Sigma-Aldrich), then administered Trypan blue solution (0.4\% in PBS i.v.; Sigma-Aldrich) and killed 10 minutes later. Uteri were dissected and assessed for clearly delineated blue bands as evidence of early implantation sites. In other mice, paraffin sections of uterus from DTtreated Cd11b-Dtr and wild-type mice, collected on day $4.5 \mathrm{pc}$ (following DT treatment on day $3.5 \mathrm{pc}$ ), were stained with $\mathrm{H} \& \mathrm{E}$ to localize implantation sites. Serial sections were examined for embryos at blastocyst stage, identified as spherical or ellipsoid bodies of approximately 100 cells with a defined inner cell mass and blastocele cavity, detectable on 4-6 serial sections.

Macrophage reconstitution. Bone marrow was harvested from the tibia and femur of adult female FVB/N mice, treated with flash lysis to remove rbc, then cultured in RPMI-1640 (Invitrogen) containing 10\% FCS, L-glutamine, and antibiotics plus 20\% L929-conditioned media for 7-10 days. Differentiation into more than $85 \% \mathrm{CD} 11 \mathrm{~b}^{+} \mathrm{F} 4 / 80^{+}$monocyte/macrophages was confirmed by flow cytometry (Supplemental Figure 2). Cd11b-Dtr mice were injected i.v. with 5-10 ' $10^{6}$ bone marrow-derived monocyte/macrophages twice, at 10 am on day $0.5 \mathrm{pc}$, and again just prior to DT administration at $12 \mathrm{pm}$ on day $3.5 \mathrm{pc}$.

Ovariectomy and induction of deciduoma. Cd11b-Dtr mice were bilaterally ovariectomized under isoflurane anesthesia. Two weeks later on day 0 , mice were primed with estradiol (100 ng s.c.; Sigma-Aldrich) daily for 2 days. Commencing on day 4 , mice were treated for 6 consecutive days (day 4-9) with $\mathrm{E}_{2}(10 \mathrm{ng})$ and $\mathrm{P}_{4}(500 \mu \mathrm{g}$; Sigma-Aldrich), to mimic the hormonal environment in early pregnancy (76). To induce deciduoma, on day 6 of the hormone replacement protocol, mice were anesthetized with avertin, and $10 \mu \mathrm{l}$ of sesame oil (Sigma-Aldrich) was injected into the uterine lumen at the distal end of the left uterine horn, close to the uterotubal junction, via a dorsal incision. The same procedure was performed on the right horn, with insertion of a sterile needle only as a sham control. Additionally, all mice were given PBS or DT ( $25 \mathrm{ng} / \mathrm{g}$ i.p.) on each of days 4,6 , and 8 of the protocol. Twenty-four hours later on day 9 , mice were killed and deciduoma formation was assessed as the relative increase in uterine horns (fold change of uterine weight equivalent to weight of left horn/weight of right horn) as well as through histochemical staining of uterine tissue sections for alkaline phoshatase activity.

Embryo collection and culture. Wild-type and Cd11b-Dtr females were mated with males of the same genotype, given DT ( $25 \mathrm{ng} / \mathrm{g}$ i.p.) on day $2.5 \mathrm{pc}$, and then 24 hours later, at 10 am on day $3.5 \mathrm{pc}$, mice were killed and the oviducts and uteri excised. Embryos were flushed and scored for developmental stage. To obtain zygotes (1-cell embryos), pre-pubertal Cd11b-Dtr females (3-4 weeks, 12-14 g) were given equine chorionic gonadatropin (Folligon; Intervet; 5 IU i.p.), followed 48 hours later by human chorionic gonadatropin (hCG) (Chorulon; Intervet; 5 IU i.p.) and mated with Cd11bDtr males. Zygotes were flushed from oviducts of mice killed at $3 \mathrm{pm}$ on day $0.5 \mathrm{pc}$. and were placed in $0.5 \mathrm{mg} / \mathrm{ml}$ of hyaluronidase (Sigma-Aldrich) for up to 1 minute to remove cumulus cells prior to culture in G1 medium (Vitrolife) with or without addition of DT $(25 \mathrm{ng} / \mathrm{ml})$. At the 8-cell stage (48 hours of culture), embryos were transferred into G2 culture medium without DT for a further 48 hours. Embryos at the 8-cell stage were flushed from the oviducts and uteri of additional superovulated Cd11b-Dtr mice on day $2.5 \mathrm{pc}$ and were cultured in G2 medium with or without addition of DT $(25 \mathrm{ng} / \mathrm{ml})$ for 72 hours. Embryos were cultured in groups of 12 to 15 in $20 \mu \mathrm{l}$ droplets under mineral oil (Sigma-Aldrich) at $37^{\circ} \mathrm{C}$ in $5 \% \mathrm{O}_{2}$ and $6 \% \mathrm{CO}_{2}$, and were scored daily for developmental stage.

In vitro luteal cell culture. Pre-pubertal Cd11b-Dtr mice were hyperstimulated with hormones as described above, then mated with male Cd11b-Dtr mice. On day 2.5 pc, ovaries were dissected in DMEM-F12 (Invitrogen) supplemented with $25 \mathrm{mM}$ HEPES, 10\% FBS, $2 \mathrm{mM}$ L-glutamine, and antibiotics (DMEM-HEPES-FBS). For each experiment, a single-cell suspension was prepared using ovaries from 6-8 mice by gentle manual disruption in a glass homogenizer. After flash lysis to remove rbc, cells were resuspended in DMEM-F12 supplemented with 10\% FCS, L-glutamine, and antibiotics at $4^{\prime} 10^{5}$ cells per well. After 24 hours, cells were washed 3 times and the media replaced; an additional 24 hours later, peritoneal macrophages $\left(6^{\prime} 10^{5}\right.$ cells) were added to the wells and allowed to adhere for 4 hours before washing 4 times to remove non-adherent cells. Elicited peritoneal macrophages were obtained from FVB/N mice given $300 \mu \mathrm{l}$ of $10 \%$ sterile thioglycollate (BD BioSciences) 4 days prior to collection. DT ( $1 \mu \mathrm{g} /$ well $)$ or vehicle (PBS) was added to wells, and supernatants were collected 48 hours later. Progesterone concentrations in supernatants were measured using enzyme-linked immunoassay (ALPCO Diagnostics).

Histology and immunohistochemistry. Ovary and uterus were collected at various time points during the pre-implantation period, each 24 hours following DT administration. For most paraffin sections, uterus and ovaries were fixed in $4 \% \mathrm{PFA}$ in PBS overnight at $4^{\circ} \mathrm{C}$ and embedded in paraffin. Tissue sections $(6 \mu \mathrm{m})$ were mounted on glass slides and blocked with $15 \%$ normal rabbit serum (NRS) plus 15\% normal mouse serum for 30 minutes. Slides were incubated at $4{ }^{\circ} \mathrm{C}$ overnight with rat anti-F4/80 (1:100; Caltag Laboratories), followed by 40 minutes at room temperature with biotinylated rabbit anti-rat IgG (1:600; Vector Laboratories), and detection used ABC Vectorstain Elite reagents (Vector Laboratories) with DAB plus $\mathrm{H}_{2} \mathrm{O}_{2}$ (SigmaFast DAB; Sigma-Aldrich). Tissue sections were counterstained with hematoxylin (Sigma-Aldrich). Images of stained sections were captured using a Hamamatsu Photonics Nanozoomer (Hamamatsu).

For fresh frozen sections, ovary and uterus were embedded in OCT compound (Tissue-Tek; Sakuta Fintek) and frozen in liquid $\mathrm{N}_{2}$ nitrogen-cooled isopentane. Cryostat sections $(6 \mu \mathrm{m})$ were mounted on glass slides and fixed in $96 \%$ ethanol ( $\mathrm{vol} / \mathrm{vol}$ ) for 10 minutes at $4^{\circ} \mathrm{C}$. To detect endothelial cells, sections were incubated with rat MTS-12 (neat hybridoma supernatant; gift from Richard Boyd, Monash University, Melbourne, Australia) (77). Reactivity was visualized using biotinylated rabbit anti-rat IgG (1:400; DakoCytomation), followed by avertin-HRP (1:400; DakoCytomation), then DAB 
plus $\mathrm{H}_{2} \mathrm{O}_{2}$. Eosinophils in uterus and ovary were detected using incubation of ethanol-fixed frozen sections with $\mathrm{DAB}$ plus $\mathrm{H}_{2} \mathrm{O}_{2}$. To quantify macrophages, 10 fields per captured image and 2 images per tissue were analyzed using Video-Pro software (Leading Edge Software). Macrophages were quantified in tissue sections as percentage of $\mathrm{F} 4 / 80$ positivity $=100^{\prime}$ (area positive F4/80 staining - area eosinophil staining) / area total staining (DAB plus hematoxylin).

To colocalize macrophages and endothelial cells, sections were incubated with Alexa Fluor 488-conjugated rat anti-F4/80 (clone BM8, 1:50; Biolegend) and Alexa Fluor 647-conjugated rat anti-CD31 (PECAM1, clone 390, 1:100; Biolegend). Lymphatic vessels were detected with rabbit polyclonal anti-LYVE1 (1:100; Millipore Corporation), followed by Alexa Fluor 594conjugated goat anti-rabbit IgG (Invitrogen). Sections were counterstained with DAPI (Molecular Probes) and mounted under coverslips using fluorescent mounting media (Dako). Immunofluorescent staining was visualized using Radiance 2100 laser scanning confocal microscopy (Olympus and BioRad). In pilot dual-labeling experiments, MTS-12 and CD31 were shown to colocalize in ovary tissue. Negative controls used isotypematched irrelevant rat or rabbit primary antibodies.

Ovaries for colocalization of F4/80 and TIE2 were recovered after perfusion fixation of FVB mice on day 2.5 pc with $4 \%$ PFA in PBS, then fixed in $4 \%$ PFA overnight at $4^{\circ} \mathrm{C}$, followed by 24 hours' incubation each in $18 \%$ sucrose and $30 \%$ sucrose in PBS. Ovaries were embedded in OCT compound and frozen in liquid $\mathrm{N}_{2}$ nitrogen-cooled isopentane. Cryostat sections $(6 \mu \mathrm{m})$ were mounted on glass slides, dried for 1 hour, and then incubated with proteinase $\mathrm{K}(1 \mu \mathrm{g} / \mathrm{ml}$; Sigma-Aldrich) for 10 minutes at room temperature. Sections were incubated with anti-TIE2 (CD202b, clone TEK4, 1:200; e-Biosciences), followed by Alexa Fluor 594-conjugated donkey anti-rat (Invitrogen), Alexa Fluor 488-conjugated rat anti-F4/80 (clone BM8, 1:50; Biolegend), and then counterstained with DAPI and visualized as described above.

H\&E staining was performed using standard protocols on paraffin sections. Alkaline phosphatase staining to detect decidual cells was performed on fresh frozen sections fixed in ethanol as described above, then incubated for 5-10 minutes with SigmaFast BCIP/NBT (5-bromo-4-chloro3-indolyl phosphate/nitro blue tetrazolium; Sigma-Aldrich) according to the manufacturer's instructions. Sections were counterstained using eosin (Sigma-Aldrich).

Flow cytometry. Wild-type and Cd11b-Dtr mice were killed 24 hours after DT administration, and ovary and uterus were excised after collection of peritoneal exudate cells (PEC) by peritoneal lavage using $5 \mathrm{ml}$ of FACS buffer (PBS containing 0.1\% BSA [Sigma-Aldrich] and $0.025 \%$ sodium azide [Sigma-Aldrich]). Uterus and ovary were trimmed of fat and chopped into small pieces prior to enzymatic digestion into single cells by incubation for 1 hour at $37^{\circ} \mathrm{C}$ in RPMI containing $10 \%$ FBS, 0.015 M HEPES (JRH Biosciences), and 2,000 $\mathrm{U} / \mathrm{ml}$ type $1 \mathrm{~A}$ collagenase from Clostridium histolyticum (Sigma-Aldrich) and $25 \mu \mathrm{g} / \mathrm{ml}$ DNase 1 (Sigma-Aldrich). Cells were passed through a $70-\mu \mathrm{m}$ nylon strainer (BD Falcon) and washed in FACS buffer. Fc receptors were blocked through incubation with anti-Fc- $\gamma$ IIR antibody $\left(10 \mu \mathrm{g} / \mathrm{ml} \mathrm{FcBlock}\right.$; BD Biosciences) for 10 minutes at $4{ }^{\circ} \mathrm{C}$. Surface markers were labeled by adding $50 \mu \mathrm{l}$ of FACS buffer containing combinations of $0.5 \mu \mathrm{g}$ each of Alexa Fluor 488-conjugated rat anti-F4/80 (clone BM8), allophycocyanin-conjugated (APC-conjugated) CD11b (clone M1/70), Alexa Fluor 488-conjugated rat anti-CD11c (clone N418), FITC-conjugated anti-CD3 (clone 17A2), phycoethrin/Cy5.5-conjugated (PE/Cy5.5-conjugated) rat anti-RB6 (LY6G/C, clone RB6-8C5), or PE/Cy7-conjugated rat anti-CD45 (clone 30-F-11) (all BD Biosciences) for 30 minutes at $4{ }^{\circ} \mathrm{C}$. Data were analyzed using FACS Canto (BD Biosciences) and FACS Diva software (version 6.0; BD Biosciences). Propidium iodide was added to exclude nonviable cells. Gates were applied to forward-scatter/side-scatter dot plots to exclude non-viable cells and cell debris.
Plasma progesterone, estradiol, and PRL analysis. Approximately $1 \mathrm{ml}$ of blood was collected through cardiac puncture in mice under deep avertin anesthesia. Plasma estradiol and progesterone concentrations were measured using commercial radioimmunoassay kits (DSL-4800 and DSL-3400, respectively) according to the manufacturer's instructions (Diagnostic Systems Laboratories). All Cd11b-Dtr and wild-type samples were measured in a single assay. The within-assay coefficients of variation were $10.0 \%$ (estradiol) and $7.0 \%$ (progesterone). The lower limits of detection were $5 \mathrm{pg} / \mathrm{ml}$ estradiol and $0.3 \mathrm{ng} / \mathrm{ml}$ progesterone. Plasma PRL was measured using a mouse enzyme-linked immunoassay (R\&D Systems). This assay was designed to detect PRL in culture supernatants, so to enable detection in plasma, standards and plasma samples were diluted in 10\% FBS in PBS as detailed in the manufacturer's instructions. Plasma from non-pregnant mice was spiked with recombinant PRL standard to ensure appropriate recovery using this method. The lower limit of detection was $100 \mathrm{pg} / \mathrm{ml}$ PRL.

Quantitative real-time PCR. Total ovarian RNA was extracted using Trizol (Invitrogen) and treated with DNase (DNA free; Ambion), then first-strand cDNA was reverse-transcribed from $3 \mu \mathrm{g}$ random hexamer-primed RNA using a Superscript-III Reverse Transcriptase kit. Primer pairs specific for published cDNA sequences were designed using Primer Express version 2 software (Applied Biosystems). For PCR, reagents were supplied in a 2' SYBR Green PCR Master Mix (Applied Biosystems), and each reaction (20 $\mu \mathrm{l}$ total) contained $3 \mu \mathrm{lcDNA}$ and $0.5 \mu \mathrm{M}$ of $5^{\prime}$ and $3^{\prime}$ primers. The following primer pairs were used to detect specific cDNAs: Actb, $5^{\prime}$-TGTGATGGTGGGTATGGGTC, 3'-ACACGCAGCTCATTGTA; Star, 5' -CCGGAGCAGAGTGGTGTCAT, 3'-TGCGATAGGACCTGGTTGATG; Cyp11a1, 5'-CACTCCTCAAAGCCAGCATCA, 3'-ACGAAGCACCAGGTCATTCAC; Hsd3b1, 5'-GGACAAAGTATTCCGACCAGAAAC, 3'-CAGGCACTGGGCATCCA; Vegfa, 5'-GCAGGCTGCTGTAACGATGA, 3'-CGCTCTGAACAAGGCTCACA; Vegfb, 5'-CCCCTGTGTCCCAGTTTGAT, 3'-TGCCCATGAGTTCCATGCT; Vegfc, 5'-AGACGTTCTCTGCCAGCAACA, 3'-CAGGCATCGGCACATGTAGTT; Figf, 5'-GTGGACCGCACATGACGTT, 3'-CAGGCTGGCTTTCTACTTGCA; Bax, 5'-GAGATGAACTGGACAGCAATATGG, 3'-GCAAAGTAGAAGAGGGCAACCA; Bcl2, 5'-GGAGAGCGTCAACAGGGAGA, 3'-CAGCCAGGAGAAATCAAACAGAG; Hif1a, 5'-CCTCCGATTTAGCATGCAGACT, 3'-GCCTTGTATGGGAGCATTAACTTC; Hsp90aa1, 5'-AATGCTTAGAACTATTTACTGAACTAGCAGAA, 3'-GTCCTCGTGAATTCCAAGCTTT; Hsp90ab1, 5'-GCGCACGCTGACTTTGGT, 3'-CCTGGAGAGCCTCCATGAAC; Akr1c18, 5'-TTTGGTCAACTTCCCATCGTC, 3'-CAAAAGCTCATTCCCTGGCT; Lhr, 5'-TAACCACCATACCAgGgAaCG, 3'-GTCGTCCCATTGAATGCATG; $P r l r$, 5'-TGCAAGCCAGACCATGGATA, 3'-GAGAGAACGGCCACAATGATC; Ptgs2 (Cox2), 5'-GCTTCGGGAGCACAACAGA, 3'-GTAACCGCTCAGGTGTTGCA; Tnfa, 5'-GTAGCCCACGTCGTAGCAAAC, 3'-CTGGCACCACTAGTTGGTTGTC; Il6, 5'-ACAACCACGGCCTTCCCTAC, 3'-TCCACGATTTCCCAGAGAACA. The negative control contained $\mathrm{H}_{2} \mathrm{O}$ substituted for cDNA. PCR amplification was performed in duplicate in an ABI Prism 7000 Sequence Detection System (Applied Biosystems). Reaction products were analyzed by dissociation curve profile and by $2 \%$ agarose gel (wt/vol) electrophoresis. Assay optimization and validation experiments were performed to define the amplification efficiency of each primer pair. Messenger RNA abundance values were normalized independently to Actb mRNA expression, and data are plotted in the figures as relative expression in arbitrary units, adjusted such that the mean of the wild-type control group is assigned a value of 100 .

Statistics. Data were assessed for normal distribution with a ShapiroWilk normality test using SPSS Statistics version 17.0 software (IBM Corporation). Normally distributed data were analyzed using ANOVA and an unpaired $t$ test. If data were not normally distributed, or if distribution could not be determined due to small sample size, data were analyzed 
using a Mann-Whitney $U$ test. Individual datum points were excluded as outliers if they were greater than 2 standard deviations from the mean. The effect of treatment group on categorical data was evaluated with the Fisher's Exact test. The difference between groups was considered statistically significant if $P<0.05$.

Study approval. This study was approved by the University of Adelaide Animal Ethics Committee and conducted in accordance with the Australian Code of Practice for the Care and Use of Animals for Scientific Purposes (7th edition; 2004).

\section{Acknowledgments}

The authors thank David Sharkey for completing the PRL ELISA and Ghafar Sarvestani for assistance with confocal microscopy. This work was supported by National Health and Medical Research
Council (Australia) (NHMRC) Program and Fellowship grants (to S.A. Robertson), an NHMRC Dora Lush Postgraduate Biomedical Scholarship (to A.S. Care), an NHMRC Early Career Research Fellowship (to K.R. Diener), an NHMRC project grant (to W.V. Ingman), and project grants from the Channel 7 Children's Research Foundation and the Clive and Vera Ramiciotti Foundation (to M.J. Jasper).

Received for publication September 14, 2012, and accepted in revised form May 9, 2013.

Address correspondence to: Sarah A. Robertson, Robinson Institute, University of Adelaide, Adelaide, SA 5005 Australia. Phone: 61.8.8303.4094; Fax: 61.8.8303.4099; E-mail: sarah.robertson@ adelaide.edu.au.
1. Gordon S. The macrophage: past, present and future. Eur J Immunol. 2007;37(suppl 1):S9-S17.

2. Sunderkotter C, Steinbrink K, Goebeler M, Bhardwaj R, Sorg C. Macrophages and angiogenesis. J Leukoc Biol. 1994;55(3):410-422.

3. Lamagna C, Aurrand-Lions M, Imhof BA. Dual role of macrophages in tumor growth and angiogenesis. J Leukoc Biol. 2006;80(4):705-713.

4. Pollard JW. Trophic macrophages in development and disease. Nat Rev Immunol. 2009;9(4):259-270.

5. Hume DA, Ross IL, Himes SR, Sasmono RT, Wells CA, Ravasi T. The mononuclear phagocyte system revisited. J Leukoc Biol. 2002;72(4):621-627.

6. Hunt JS, Robertson SA. Uterine macrophages and environmental programming for pregnancy success. J Reprod Immunol. 1996;32(1):1-25.

7. Cohen PE, Nishimura K, Zhu L, Pollard JW. Macrophages: important accessory cells for reproductive function. J Leukoc Biol. 1999;66(5):765-772.

8. De M, Wood GW. Influence of oestrogen and progesterone on macrophage distribution in the mouse uterus. J Endocrinol. 1990;126(3):417-424.

9. Robertson SA, Mau VJ, Tremellen KP, Seamark RF. Role of high molecular weight seminal vesicle proteins in eliciting the uterine inflammatory response to semen in mice. J Reprod Fertil. 1996;107(2):265-277.

10. Jasper MJ, Care AS, Sullivan B, Ingman WV, Aplin JD, Robertson SA. Macrophage-derived LIF and IL1B regulate alpha(1,2)fucosyltransferase 2 (Fut2) expression in mouse uterine epithelial cells during early pregnancy. Biol Reprod. 2011;84(1):179-188.

11. Fest $S$, et al. Trophoblast-macrophage interactions: a regulatory network for the protection of pregnancy. Am J Reprod Immunol. 2007;57(1):55-66.

12. Wu R, Van der Hoek KH, Ryan NK, Norman RJ, Robker RL. Macrophage contributions to ovarian function. Hum Reprod Update. 2004;10(2):119-133.

13. Brannstrom M, Mayrhofer G, Robertson SA. Localization of leukocyte subsets in the rat ovary during the periovulatory period. Biol Reprod. 1993;48(2):277-286.

14. Van der Hoek KH, Maddocks S, Woodhouse CM, van Rooijen N, Robertson SA, Norman RJ. Intrabursal injection of clodronate liposomes causes macrophage depletion and inhibits ovulation in the mouse ovary. Biol Reprod. 2000;62(4):1059-1066.

15. Turner EC, et al. Conditional ablation of macrophages disrupts ovarian vasculature. Reproduction 2011;141(6):821-831.

16. Cohen PE, Zhu L, Pollard JW. Absence of colony stimulating factor- 1 in osteopetrotic (csfmop/csfmop) mice disrupts estrous cycles and ovulation. Biol Reprod. 1997;56(1):110-118.

17. Brannstrom M, Giesecke L, Moore IC, van den Heuvel CJ, Robertson SA. Leukocyte subpopulations in the rat corpus luteum during pregnancy and pseudopregnancy. Biol Reprod. 1994;50(5):1161-1167.

18. Brannstrom M, Pascoe V, Norman RJ, McClure
N. Localization of leukocyte subsets in the follicle wall and in the corpus luteum throughout the human menstrual cycle. Fertil Steril. 1994;61(3):488-495.

19. Kirsch TM, Friedman AC, Vogel RL, Flickinger GL. Macrophages in corpora lutea of mice: characterization and effects on steroid secretion. Biol Reprod. 1981;25(3):629-638.

20. Nelson SE, McLean MP, Jayatilak PG, Gibori G. Isolation, characterization, and culture of cell subpopulations forming the pregnant rat corpus luteum. Endocrinology. 1992;130(2):954-966.

21. Wiktor-Jedrzejczak W, et al. Total absence of colony-stimulating factor 1 in the macrophage-deficient osteopetrotic (op/op) mouse. Proc Natl Acad SciU S A. 1990;87(12):4828-4832.

22. Pollard JW, Hunt JS, Wiktor-Jedrzejczak W, Stanley ER. A pregnancy defect in the osteopetrotic (op/op) mouse demonstrates the requirement for CSF-1 in female fertility. Dev Biol. 1991;148(1):273-283.

23. Cohen PE, Zhu L, Nishimura K, Pollard JW. Colony-stimulating factor 1 regulation of neuroendocrine pathways that control gonadal function in mice. Endocrinology. 2002;143(4):1413-1422.

24. Pollard JW, Lin EY, Zhu L. Complexity in uterine macrophage responses to cytokines in mice. Biol Reprod. 1998;58(6):1469-1475

25. Robertson SA, Allanson M, Mau VJ. Molecular regulation of uterine leukocyte recruitment during early pregnancy in the mouse. Placenta. 1998;19(suppl 1):101-119.

26. Duffield JS, et al. Selective depletion of macrophages reveals distinct, opposing roles during liver injury and repair. J Clin Invest. 2005;115(1):56-65.

27. Robertson SA, Mau VJ, Young IG, Matthaei KI. Uterine eosinophils and reproductive performance in interleukin 5-deficient mice. J Reprod Fertil. 2000;120(2):423-432.

28. Cailhier JF, et al. Conditional macrophage ablation demonstrates that resident macrophages initiate acute peritoneal inflammation. J Immunol. 2005;174(4):2336-2342.

29. Niswender GD, Juengel JL, Silva PJ, Rollyson MK, McIntush EW. Mechanisms controlling the function and life span of the corpus luteum. Physiol Rev. 2000;80(1):1-29.

30. Stocco C, Telleria C, Gibori G. The molecular control of corpus luteum formation, function, and regression. Endocr Rev. 2007;28(1):117-149.

31. Telleria CM, Ou J, Sugino N, Ferguson S, Gibori G. The expression of interleukin- 6 in the pregnant rat corpus luteum and its regulation by progesterone and glucocorticoid. Endocrinology. 1998;139(8):3597-3605

32. Abdo M, Hisheh S, Dharmarajan A. Role of tumor necrosis factor-alpha and the modulating effect of the caspases in rat corpus luteum apoptosis. Biol Reprod. 2003;68(4):1241-1248.

33. Stocco CO, Zhong L, Sugimoto Y, Ichikawa A,
Lau LF, Gibori G. Prostaglandin F2alpha-induced expression of 20alpha-hydroxysteroid dehydrogenase involves the transcription factor NUR77. J Biol Chem. 2000;275(47):37202-37211.

34. Pucci $F$, et al. A distinguishing gene signature shared by tumor-infiltrating Tie2-expressing monocytes, blood "resident" monocytes, and embryonic macrophages suggests common functions and developmental relationships. Blood. 2009;114(4):901-914.

35. Ferrara N, Davis-Smyth T. The biology of vascular endothelial growth factor. Endocr Rev. 1997;18(1):4-25.

36. Reynolds LP, Killilea SD, Redmer DA. Angiogenesis in the female reproductive system. FASEB J. 1992;6(3):886-892.

37. Kizuka F, et al. Involvement of bone marrowderived vascular progenitor cells in neovascularization during formation of the corpus luteum in mice. Biol Reprod. 2012;87(3):55.

38. Lydon JP, et al. Mice lacking progesterone receptor exhibit pleiotropic reproductive abnormalities. Genes Dev. 1995;9(18):2266-2278.

39. De Palma M, Murdoch C, Venneri MA, Naldini L, Lewis CE. Tie2-expressing monocytes: regulation of tumor angiogenesis and therapeutic implications. Trends Immunol. 2007;28(12):519-524.

40. Felcht $M$, et al. Angiopoietin-2 differentially regulates angiogenesis through TIE2 and integrin signaling. J Clin Invest. 2012;122(6):1991-2005.

41. He H, et al. Endothelial cells provide an instructive niche for the differentiation and functional polarization of M2-like macrophages. Blood. 2012;120(15):3152-3162.

42. Shirasuna K, Shimizu T, Matsui M, Miyamoto A. Emerging roles of immune cells in luteal angiogenesis. Reprod Fertil Dev. 2013;25(2):351-361.

43. Grunewald M, et al. VEGF-induced adult neovascularization: recruitment, retention, and role of accessory cells. Cell. 2006;124(1):175-189.

44. Cursiefen C, et al. VEGF-A stimulates lymphangiogenesis and hemangiogenesis in inflammatory neovascularization via macrophage recruitment. J Clin Invest. 2004;113(7):1040-1050.

45. Phillips HS, Hains J, Leung DW, Ferrara N. Vascular endothelial growth factor is expressed in rat corpus luteum. Endocrinology. 1990;127(2):965-967.

46. Wulff C, Wilson H, Largue P, Duncan WC, Armstrong DG, Fraser HM. Angiogenesis in the human corpus luteum: localization and changes in angiopoietins, Tie-2, and vascular endothelial growth factor messenger ribonucleic acid. J Clin Endocrinol Metab. 2000;85(11):4302-4309.

47. Wulff C, Wilson H, Rudge JS, Wiegand SJ, Lunn SF, Fraser HM. Luteal angiogenesis: prevention and intervention by treatment with vascular endothelial growth factor trap(A40). J Clin Endocrinol Metab. 2001;86(7):3377-3386

48. Fraser HM, Wilson H, Wulff C, Rudge JS, Wiegand 
SJ. Administration of vascular endothelial growth factor Trap during the 'post-angiogenic' period of the luteal phase causes rapid functional luteolysis and selective endothelial cell death in the marmoset. Reproduction. 2006;132(4):589-600.

49. Ferrara N, et al. Vascular endothelial growth factor is essential for corpus luteum angiogenesis. Nat Med. 1998;4(3):336-340.

50. Granata F, et al. Production of vascular endothelial growth factors from human lung macrophages induced by group IIA and group $\mathrm{X}$ secreted phospholipases A2. J Immunol. 2010; 184(9):5232-5241.

51. Schoppmann SF, et al. Tumor-associated macrophages express lymphatic endothelial growth factors and are related to peritumoral lymphangiogenesis. Am J Pathol. 2002;161(3):947-956.

52. Achen MG, et al. Vascular endothelial growth factor $\mathrm{D}$ (VEGF-D) is a ligand for the tyrosine kinases VEGF receptor 2 (Flk1) and VEGF receptor 3 (Flt4). Proc Natl Acad Sci U S A. 1998;95(2):548-553.

53. Joukov V, et al. Proteolytic processing regulates receptor specificity and activity of VEGF-C. EMBOJ. 1997;16(13):3898-3911.

54. Cao Y, et al. Vascular endothelial growth factor C induces angiogenesis in vivo. Proc Natl Acad SciUS A. 1998;95(24):14389-14394.

55. Rissanen TT, et al. VEGF-D is the strongest angiogenic and lymphangiogenic effector among VEGFs delivered into skeletal muscle via adenoviruses. Circ Res. 2003;92(10):1098-1106.

56. Tammela T, et al. VEGFR-3 controls tip to stalk conversion at vessel fusion sites by reinforcing Notch signalling. Nat Cell Biol. 2011;13(10):1202-1213.

57. Staples LD, Fleet IR, Heap RB. Anatomy of the utero-ovarian lymphatic network and the composition of afferent lymph in relation to the establishment of pregnancy in the sheep and goat. J Reprod Fertil.
1982;64(2):409-420.

58. Alitalo K, Tammela T, Petrova TV. Lymphangiogenesis in development and human disease. Nature. 2005;438(7070):946-953.

59. Senger D, Galli S, Dvorak A, Perruzzi C, Harvey V, Dvorak H. Tumor cells secrete a vascular permeability factor that promotes accumulation of ascites fluid. Science. 1983;219(4587):983-985.

60. Erlebacher A, Zhang D, Parlow AF, Glimcher LH Ovarian insufficiency and early pregnancy loss induced by activation of the innate immune system. J Clin Invest. 2004;114(1):39-48.

61. Friedman A, Weiss S, Levy N, Meidan R. Role of tumor necrosis factor alpha and its type I receptor in luteal regression: induction of programmed cell death in bovine corpus luteum-derived endothelial cells. Biol Reprod. 2000;63(6):1905-1912.

62 . Rueda BR, et al. Increased bax and interleukin1beta-converting enzyme messenger ribonucleic acid levels coincide with apoptosis in the bovine corpus luteum during structural regression. Biol Reprod. 1997;56(1):186-193.

63. Khanna A, Aten RF, Behrman HR. Heat shock protein-70 induction mediates luteal regression in the rat. Mol Endocrinol. 1995;9(11):1431-1440.

64. Tanaka M, et al. Participation of reactive oxygen species in PGF2alpha-induced apoptosis in rat luteal cells. J Reprod Fertil. 2000;120(2):239-245.

65 . Jasper MJ, Robertson SA, Van der Hoek KH, Bonello N, Brannstrom M, Norman RJ. Characterization of ovarian function in granulocyte-macrophage colony-stimulating factor-deficient mice. Biol Reprod. 2000;62(3):704-713.

66. Ingman WV, Robker RL, Woittiez K, Robertson SA. Null mutation in transforming growth factor beta1 disrupts ovarian function and causes oocyte incompetence and early embryo arrest. Endocrinology. 2006;147(2):835-845.
67. Duffield JS, et al. Conditional ablation of macrophages halts progression of crescentic glomerulonephritis. Am J Pathol. 2005;167(5):1207-1219.

68. Mirza R, DiPietro LA, Koh TJ. Selective and specific macrophage ablation is detrimental to wound healing in mice. Am J Pathol. 2009;175(6):2454-2462.

69. Chua AC, Hodson LJ, Moldenhauer LM, Robertson SA, Ingman WV. Dual roles for macrophages in ovarian cycle-associated development and remodelling of the mammary gland epithelium. Development. 2010;137(24):4229-4238.

70. Hinney B, Henze C, Kuhn W, Wuttke W. The corpus luteum insufficiency: a multifactorial disease. J Clin Endocrinol Metab. 1996;81(2):565-570.

71. Pritts EA, Atwood AK. Luteal phase support in infertility treatment: a meta-analysis of the randomized trials. Hum Reprod. 2002;17(9):2287-2299.

72. Coomarasamy A, Truchanowicz EG, Rai R. Does first trimester progesterone prophylaxis increase the live birth rate in women with unexplained recurrent miscarriages? BMJ. 2011;342:d1914.

73. Chinetti-Gbaguidi G, Staels B. Macrophage polarization in metabolic disorders: functions and regulation. Curr Opin Lipidol. 2011;22(5):365-372.

74. Lumeng CN, Bodzin JL, Saltiel AR. Obesity induces a phenotypic switch in adipose tissue macrophage polarization. J Clin Invest. 2007;117(1):175-184.

75. Joachim $\mathrm{R}$, et al. The progesterone derivative dydrogesterone abrogates murine stress-triggered abortion by inducing a Th2 biased local immune response. Steroids. 2003;68(10-13):931-940.

76. Finn CA, Martin L. Endocrine control of the timing of endometrial sensitivity to a decidual stimulus. Biol Reprod. 1972;7(1):82-86.

77. Tucek CL, Godfrey DI, Boyd RL. Five novel antigens illustrate shared phenotype between mouse thymic stromal cells, thymocytes, and peripheral lymphocytes. Int Immunol. 1992;4(9):1021-1030. 